\title{
Numerical Relativity with the Conformal Field Equations
}

\author{
Sascha Husa \\ Max-Planck-Institut für Gravitationsphysik, 14476 Golm, Germany
}

\begin{abstract}
I discuss the conformal approach to the numerical simulation of radiating isolated systems in general relativity. The method is based on conformal compactification and a reformulation of the Einstein equations in terms of rescaled variables, the so-called "conformal field equations" developed by Friedrich. These equations allow to include "infinity" on a finite grid, solving regular equations, whose solutions give rise to solutions of the Einstein equations of (vacuum) general relativity. The conformal approach promises certain advantages, in particular with respect to the treatment of radiation extraction and boundary conditions. I will discuss the essential features of the analytical approach to the problem, previous work on the problem - in particular a code for simulations in $3+1$ dimensions, some new results, open problems and strategies for future work.
\end{abstract}

\section{Introduction}

In order to understand the physical content of the theory of general relativity, it is desirable to both mathematically understand its solutions and observationally understand the physical phenomena for which the theory is relevant. The latter effort typically requires predictions from the theory, both qualitative and quantitative - such as gravitational wave templates or binary pulsar deceleration parameters. The lack of genericity in available exact solutions then naturally leads to the use of approximation methods such as post-Newtonian approximations, perturbation theory or numerical analysis, which allows very general non-perturbative approximations. Concrete solutions do however also play an important role in the quest for a mathematical understanding of the solution space. The experience gained from such solutions can suggest theorems, test conjectures, or lead to the discovery of previously unknown phenomena. For some particularly interesting examples see [1], [2] or [3]. The construction and study of solutions, be it with approximate or exact methods, obviously profits from a sound mathematical basis in the form of well-posed equations, analytic estimates and the likes. Eventually - hopefully - it will also profit from observational evidence!

In the following I will discuss a particular approach to the numerical solution of the Einstein field equations, which addresses the problems associated with the treatment of asymptotic regions by conformal compactification. The interest in asymptotic regions is rooted in the problem of describing isolated systems. Physical intuition suggests that many astrophysical processes (whether 
they are of actual astrophysical relevance or rather hypothetical) should essentially be independent of the large-scale structure of the universe, or, say, the local galaxy. The idealization of an isolated system, where the geometry approaches a Minkowski geometry at large distances, thus forms the basis for the general-relativistic analysis of processes which are essentially of non-cosmological nature. The mathematical formalization of the physical idea of isolated systems is the concept of asymptotically flat spacetimes. This formalization is already nontrivial, due to the lack of a preferred background geometry or coordinate system - with respect to which one could define "distance" and the appropriate limits. Conformal compactification, however, renders possible a discussion of asymptotically flat spacetimes in terms of local differential geometry. In this approach, pioneered by Penrose [4], an unphysical Lorentzian metric $g_{a b}$ is introduced on an unphysical manifold $\mathcal{M}$ which gives rise to the physical metric $\tilde{g}_{\tilde{a} b}$ by the rescaling $\tilde{g}_{a b}=\Omega^{-2} g_{a b}$. The physical manifold $\tilde{\mathcal{M}}$ is then given by $\tilde{\mathcal{M}}=\{p \in \mathcal{M} \mid \Omega(p)>0\}$. In this picture physical "infinity" corresponds to a three-dimensional boundary of a four-dimensional region in $\mathcal{M}$, defined by $\Omega=0$. Limiting procedures and approximations can thus be replaced by local differential geometry on the boundary.

In gravitational theory, quantities such as the total mass, (angular) momentum or emitted gravitational radiation can only consistently be defined at "infinity". In the conformal approach the unambiguous extraction of gravitational waves from a numerical spacetime is straightforward. In the "traditional" approach to dealing with asymptotic falloff in numerical relativity, where one introduces an arbitrary spatial cutoff, matters are much more complicated and ambiguities are introduced which one would have to get rid off by complicated limiting procedures. Without at least being able to define a clean concept of radiation leaving or entering a system, it is furthermore very hard to define physically realistic and consistent boundary conditions at finite distance. The traditional approach is thus not completely satisfactory both from a mathematical but also from a practical point of view. Here we discuss the principal ideas of the envisioned "conformal cure", the technical and conceptual problems associated with it and the current status of this approach.

It is easy to see that the conformal cure cannot be straightforward, by writing Einstein's vacuum equations in terms of $\Omega$ and $g_{a b}$ :

$$
\tilde{G}_{a b}\left[\Omega^{-2} g_{a b}\right]=G_{a b}\left[g_{a b}\right]+\frac{2}{\Omega}\left(\nabla_{a} \nabla_{b} \Omega+g_{a b} \nabla_{c} \nabla^{c} \Omega\right)+\frac{3}{\Omega^{2}} g_{a b}\left(\nabla_{c} \Omega\right) \nabla^{c} \Omega .
$$

This expression is singular for $\Omega=0$, multiplication by $\Omega^{2}$ also does not help here because then the principal part of the partial differential equations encoded in $G_{a b}$ would degenerate at $\Omega=0$. The conformal compactification approach thus cannot be carried to the level of the field equations in a straightforward way. This step however has been achieved by Friedrich, who has developed a judicious reformulation of the equations [5-9].

These conformal field equations are regular equations for $g_{a b}$ and certain additional independent variables. 
In analytical work, such global methods have proven to provide essential simplifications leading to new results and insights. Already by providing a different point of view on some of the essential problems in numerical relativity, the conformal picture is quite helpful and can stimulate new ideas. Certainly, we desire more - to make this approach also a practical tool. There is significant hope, that global methods will eventually show advantages for practical numerical work and, despite the small number of researchers involved so far (may there be more!), some significant progress in this direction has been made.

In the present chapter I will try to sketch the present status of the quest for the conformal cure and discuss some important open questions. We will start with a brief introduction of the concepts of asymptotic flatness in terms of conformal compactification in Sect. 2, highlighting some important features of "future null infinity" and then discuss the conformal field equations. In Sect. 3 I will discuss some explicit examples of compactifying Minkowski spacetime, both to paint a more concrete picture of our scenario and to set the arena for some numerical code tests. Sect. 4 contains a brief overview of the history of numerical work on the conformal field equations, leading to a description of a 3D code written by Hübner [10-13]. New results from 3D calculations performed with this code will be presented in Sect. 5 and a discussion will be given in Sect. 6 , concluding with a roadmap for future work.

\section{Compactification and the Mathematical Description of Isolated Systems}

The material in this section is intended to present some essential ideas in a condensed form. The reader should be aware that I am not doing justice here to subtleties and long history of the mathematical description of isolated systems in general relativity - rather this section intends to motivate to look into more complete reviews such as [14-16].

\subsection{Asymptotic Flatness and Compactification}

As noted above the formulation of the concept of asymptotic flatness is far from straightforward in GR, due to the absence of a background metric or preferred coordinate system, in terms of which falloff rates can be specified. A resolution of this problem is provided by a definition of asymptotic flatness, where, after a suitable conformal rescaling of the metric, "points at infinity" are added to the manifold. One thus works on a compactified auxiliary manifold and local differential geometry can be used to study the asymptotic properties of the gravitational field. We will give a simple definition of asymptotic flatness here, which for our purposes catches all essential features. For alternative definitions and more detailed explanations compare for example $[4,14,15,17]$.

Definition 1 (asymptotic simplicity)

A smooth spacetime $\left(\tilde{\mathcal{M}}, \tilde{g}_{a b}\right)$ is called asymptotically simple, if there exists another smooth spacetime $\left(\mathcal{M}, g_{a b}\right)$ and a scalar function $\Omega$ such that: 
1. $\tilde{\mathcal{M}}$ is an open submanifold of $\mathcal{M}$ with smooth boundary $\partial \tilde{\mathcal{M}}=\mathscr{I}$ (Scri).

2. $g_{a b}=\Omega^{2} \tilde{g}_{a b}$ on $\tilde{\mathcal{M}}$, with $\Omega>0$ on $\tilde{\mathcal{M}}, \Omega=0$ on $\mathscr{I}$ and $\nabla_{a} \Omega \neq 0$ on $\mathscr{I}$.

3. Every null geodesic in $\tilde{\mathcal{M}}$ acquires two end points on $\mathscr{I}$.

Definition 2 (asymptotic flatness)

Asymptotically simple spacetimes are called asymptotically flat if their Ricci tensor $\tilde{R}_{a b}$ vanishes in a neighbourhood of $\mathscr{I}$.

Examples of asymptotically simple spacetimes, which are not asymptotically flat are the de Sitter and anti-de Sitter solutions. Correspondingly to asymptotically flat spacetimes one can consider asymptotically de Sitter and anti-de Sitter spacetimes. Note that the completeness condition 3 in Def. 1, which ensures that the entire boundary is included, excludes black-hole spacetimes. For modifications to weaken condition 3 , thus allowing black holes, see the definitions of [18] or [15]. For example, the definition of weak asymptotic simplicity [18] requires condition 3 to hold only in a neighbourhood of $\mathscr{I}$. See e.g. [15] for a discussion of asymptotic flatness at spacelike infinity (i.e. the part of infinity which is reached along spacelike geodesics) versus null infinity (i.e. the part of infinity which is reached along null curves). The notion of asymptotic flatness at timelike infinity does not make much sense in a general situation, because then all energy would have to be radiated away, leaving only flat space behind - excluding black holes or "stars". For weak data however, in vacuum say, where all radiation eventually disperses, once expects asymptotic flatness to hold also at timelike infinity, this issue will be discussed below in application to concrete spacetimes.

The notion of asymptotic flatness of isolated systems turns out to be intimately related to the possibility of defining the total energy-momentum for such systems in general relativity - remember that no well-defined local energy density of the gravitational field is known (compare e.g. Sect. 11.2 of the textbook of Wald [15]). However, total energy-momentum quantities, which transform as a 4 -vector under asymptotic Lorentz transformations, can be assigned to null and spatial infinity of asymptotically flat spacetimes. If a manifold has more than one asymptotically flat end, e.g. in the presence of wormholes of the EinsteinRosen-bridge type, then different energy-momenta can be associated with each of these asymptotic regions.

The expression for the energy-momentum four-vector at spatial infinity has been given first by Arnowitt, Deser and Misner in 1962 [19] in the context of the Hamiltonian formalism and is usually called the ADM momentum, the time component being called ADM mass. The ADM energy corresponds to the energy of some Cauchy surface, i.e. a snapshot of the spacetime at some fixed time. It is a constant of motion and can therefore be expressed in terms of the initial data on an asymptotically flat Cauchy hypersurface.

The expression for the energy-momentum at null infinity, usually referred to as the Bondi energy-momentum, can be associated with a fixed retarded time, i.e. some asymptotically null surface. The decrease of this quantity measures 
the energy-momentum carried away by gravitational radiation. For a brief introduction and references to original work on different definitions of the Bondi mass see e.g. the textbook of Wald [15]. The formulation most appropriate for usage in numerical codes based on the conformal field equations was given by Penrose [4], and defines the Bondi mass in terms of the behaviour of certain projections of the Weyl tensor at $\mathscr{I}^{+}$and the shear of the outgoing congruence of null geodesics orthogonal to $\mathscr{I}$ in the gauge defined below by (2). It was already shown in 1962 by Bondi, van der Burg and Metzner [20] that the Bondi mass $M_{\mathrm{B}}$ can only decrease with time: gravitational radiation always carries positive energy away from a radiating system. Note that this means in particular, that while compactification at spatial infinity would lead to a "piling up" of waves, at $\mathscr{I}^{+}$this effect does not appear. In the compactified picture the waves leave the physical spacetime through the boundary $\mathscr{I}^{+}$.

A fundamental issue of general relativity is the positivity of the ADM and Bondi energies. Although it is trivial to write down a metric with negative mass if no conditions on the energy-momentum tensor are imposed, for reasonable matter fields with nonnegative energy density (thus satisfying the dominant energy condition), non-negativity of the ADM and Bondi energies is expected on physical grounds: if the energy of an isolated system could be negative, it would most likely be unstable and decay to lower and lower energies. Indeed, a proof of the positive definiteness of the ADM energy has been given in 1979 by Schoen and Yau [21] (several simplified proofs have been given later) and was extended to the Bondi mass in 1982 by Horowitz and Perry [22].

\subsection{What is $\mathscr{I}$ ?}

We will now have a closer look at $\mathscr{I}$ and discuss some of its features, which will allow us to understand the basic ideas of radiation extraction and help us to understand some issues related with choosing boundary conditions for numerical solutions of the conformal field equations.

Looking at (1) and multiplying by $\Omega^{2}$, one can see that for a vacuum spacetime, $\tilde{G}_{a b}=0,\left(\nabla_{c} \Omega\right) \nabla^{c} \Omega=0$ at $\mathscr{I}$, which thus must consist of null surfaces. In fact, one can then prove (see e.g. [17]), that

1. $\mathscr{I}$ has two connected components, each with topology $S^{2} \times \mathbb{R}$.

2. The connected components of $\mathscr{I}$ are smooth null hypersurfaces in $\mathcal{M}$ and as such are generated by null geodesics.

3. The congruence of null geodesic generators of $\mathscr{I}$ is shear free.

The two connected components are called future null infinity $\left(\mathscr{I}^{+}\right)$and past null infinity $\left(\mathscr{I}^{-}\right)$and provide the future and past endpoints for null geodesics in $\tilde{\mathcal{M}}$. In a naive picture they could be viewed as emanating from a point $i^{0}$ 
which represents spatial infinity ${ }^{1}$. These features will become more graphic when dealing with explicit examples below.

Note that there is gauge freedom in the choice of the conformal factor: one is free to rescale the conformal factor $\Omega$ by some $\omega>0$ such that $\hat{\Omega}=\omega \Omega$, $\hat{g}_{a b}=\omega^{2} g_{a b}=\hat{\Omega}^{2} \tilde{g}_{a b}$. It is an interesting exercise (see Sect. 11.1 of [15]) to prove that outside any neighbourhood of $i^{0}-$ on $\mathscr{I}^{+}$say - one can always use this conformal gauge freedom to achieve

$$
\hat{\nabla}_{a} \hat{\nabla}_{b} \hat{\Omega}=0 \quad \text { on } \mathscr{I}^{+},
$$

where $\hat{\nabla}_{a}$ is the derivative operator compatible with the metric $\hat{g}_{a b}$. This conformal gauge implies, that the null tangent $n^{a}=\hat{g}^{a b} \nabla_{b} \hat{\Omega}$ to the null geodesic generators of $\mathscr{I}$ satisfies the affinely parameterized geodesic equation,

$$
n^{a} \hat{\nabla}_{a} \hat{n}^{b}=0 .
$$

Consequently, expansion of the generators of $\mathscr{I}$ vanishes in addition to the shear and twist $\left(n_{a}\right.$ is a gradient). Using the remaining gauge freedom of $\omega$, we can choose coordinates such that the metric on $\mathscr{I}$ takes the form

$$
\left.\mathrm{d} \hat{s}^{2}\right|_{\mathscr{I}+}=2 \mathrm{~d} \Omega \mathrm{d} u+\mathrm{d} \theta^{2}+\sin ^{2} \theta \mathrm{d} \phi^{2},
$$

where $u$ is the affine parameter of the null geodesic generators, scaled such that $n^{a} \hat{\nabla}_{a} u=1$ (see e.g. Chap. 11 of [15]). The cuts ${ }^{2}$ of $\mathscr{I}$ of constant $u$ thus become metric spheres. The coordinate $u$ is generally known as Bondi parameter or Bondi time. The conformal gauge (2) and the coordinates (4) prove very useful in the analysis of the geometry in a neighbourhood of $\mathscr{I}$ - in particular for the extraction of radiation. The existence of a natural time coordinate (at least up to affine transformations along each generator) is very interesting for numerical applications, where at least asymptotically one can get rid of much of the slicing arbitrariness of the interior region. It is nontrivial but rather straightforward to actually (numerically) find this gauge of $\mathscr{I}^{+}$, which is also required by the standard formulas to compute the energy-momentum at $\mathscr{I}^{+}$and the emitted radiation - to be given below.

Before discussing how to compute the radiation, it is useful to idealize a detector (here I will follow the discussion in [24]). In physical space - far away from the sources - we could think of a detector as a triad of spacelike unit vectors attached to the worldline of some (timelike) observer. Let us further assume for simplicity that the observer moves along a timelike geodesic parametrized by proper time and that the triad is transported by Fermi-Walker transport. It is not hard to show - see Frauendiener [24], that taking the appropriate limit in the compactified spacetime, the observer worldline converges to a null geodesic

\footnotetext{
${ }^{1}$ The structure of $i^{0}$ is however quite subtle, significant progress toward its understanding in terms of the field equations has recently been achieved by Friedrich $[8,23]$

${ }^{2}$ A cut of $\mathscr{I}$ is a two-dimensional spacelike cross section of $\mathscr{I}$ which meets every generator once.
} 
generator of $\mathscr{I}^{+}$. Taking the limit along a Cauchy surface it converges to the point $i^{0}$, where one could naively expect an observer to end up when shifted to larger and larger distances (this limit is however not appropriate in the context of computing the radiation). Furthermore, the proper time parameter of the observer converges to Bondi time. The arbitrariness of boosting the observers is reflected in the affine freedom of choosing the Bondi parameter at $\mathscr{I}$. The description of $\mathscr{I}^{+}$thus could be condensed into the statement that it idealizes $u s$ - the observers of astrophysical phenomena happening far away. By working with the idealization, the approximations and ambiguities associated with detectors at a finite distance have transformed into a surprisingly simple geometric picture! Note that this simplification has to be taken with the typical care required in the treatment of idealizations in (theoretical) physics: under practical circumstances, e.g. computing the actual signal at a gravitational wave detector, $\mathscr{I}$ more realistically corresponds to an observer that is sufficiently far way from the source to treat the radiation linearly, but not so far away that cosmological effects have to be taken into account. In order to compute the detected signal in a realistic application, cosmological data and the fact that an earthbound detector moves in a complicated way relative to the source have to be considered.

We will next discuss a "detector-frame" adapted to $\mathscr{I}^{+}{ }_{-}$the commonly used Bondi frame. For a much more complete discussion of Bondi-systems, see e.g. the excellent review by Newman and Tod [14]. There a characteristic framework is used to set up the Bondi frame in a whole neighbourhood of $\mathscr{I}^{+}$, which is necessary to compute derivatives, entering e.g. the definition of the spin coefficient $\sigma$ defined below in (5). In the current approach, the Bondi system is only defined at $\mathscr{I}^{+}$: initial data can be set up, such that all necessary quantities can be propagated along the generators of $\mathscr{I}^{+}[25]$.

With $\mathscr{I}^{+}$being a null surface, it is most natural to use a null frame, consisting of 2 null vectors and 2 spacelike vectors $x^{a}, y^{a}$, which can be considered as the idealizations of the arms of an interferometric gravitational wave detector. The vectors $x^{a}, y^{a}$ are commonly treated in the form of two complex null vectors $m^{a}, \bar{m}^{a}$, with

$$
m^{a}=x^{a}+i y^{a}, \quad m^{a} \bar{m}_{a}=1,
$$

where $x^{a}$ and $y^{a}$ are real vectors tangent to the cuts of $\mathscr{I}^{+}$. The null vectors are taken as the affine tangent $n^{a}$ and $l_{a}=\hat{\nabla}_{a} u$, which satisfy

$$
n^{a} l_{a}=-1 \text {. }
$$

The tetrad vectors $l_{a}, m^{a}$ and $\bar{m}^{a}$ are parallely propagated along the generators, which yields transport equations that define them on all of $\mathscr{I}^{+}$once initial values are chosen.

The Bondi-mass can then be computed in terms of the spin-coefficient $\sigma$ and the rescaled Weyl tensor components $\psi_{2}$ and $\psi_{4}$ :

$$
\begin{aligned}
& \sigma=\hat{g}_{a b} l^{a} m^{c} \hat{\nabla}_{c} m^{b}, \\
& \psi_{2}=\hat{d}_{a b c d} l^{a} m^{b} \bar{m}^{c} n^{d}, \\
& \psi_{4}=\hat{d}_{a b c d} n^{a} \bar{m}^{b} n^{c} \bar{m}^{d} .
\end{aligned}
$$


In terms of these quantities the Bondi mass can be defined as

$$
M_{\mathrm{B}}=-\frac{\sqrt{A}}{\sqrt{4 \pi}^{3}} \int\left(\psi_{2}+\sigma \dot{\bar{\sigma}}\right) \mathrm{d} A,
$$

the outgoing radiation can be computed to be

$$
\dot{M}_{\mathrm{B}}=-\frac{\sqrt{A}}{\sqrt{4 \pi}^{3}} \int(\dot{\sigma} \dot{\bar{\sigma}}) \mathrm{d} A,
$$

where $A$ is the area of the cuts of $\mathscr{I}^{+}$and $\dot{f}=n^{a} \hat{\nabla}_{a} f=\partial_{u} f$. Furthermore,

$$
\ddot{\sigma}=-\bar{\psi}_{4}
$$

can be used to evolve $\sigma$, where both $\sigma$ and $\dot{\sigma}$ can be computed on the initial slice.

This procedure has been implemented by Hübner and Weaver [25] for 2D codes and the 3D code used to obtain the results in Sect. 5 and has been tested and proven accurate for several types of spacetimes [25]. Frauendiener describes his implementation and some results in [26]. There are two essential problems in these implementations: first of all, the gauge conditions will not usually result in a slicing of $\mathscr{I}^{+}$by cuts of constant Bondi time $u$. This means that interpolation has to be used between different slices of the numerical evolution. Second, in those formulations of the conformal field equations that have so far been used in numerical implementations, the conformal factor $\Omega$ is an evolution variable and not specified a priori, $\mathscr{I}$ will in general not be aligned with grid points. This results in further technical complications and an additional need for interpolation. When dealing with the physically interesting case of a $\mathscr{I}^{+}$of spherical topology, at least two patches have to be used to represent the Bondi tetrad $\left(l_{a}, n_{a}, m_{a}, \bar{m}_{a}\right)$. Frauendiener has achieved to control the movement of $\mathscr{I}^{+}$through the grid by the gauge choice for his formulation [26], in particular the shift vector can be chosen such that $\mathscr{I}$ does not change its coordinate location.

\subsection{The Conformal Field Equations}

Several formulations of the conformal field equations are available, the main difference being whether the conformal factor $\Omega$ can be specified a priori or is determined as a variable by the equations. In the original formulation $[5,6]$ and its descendants $[7,10,27] \Omega$ (and derivatives) are evolved as dependent variables. All existent numerical codes are based on equations of this type. A later version of the equations allows to fix $\Omega$ a priori and has been used to develop a new treatment of spatial infinity $i^{0}[8,23,9]$. However, the formulation and treatment of these equations is more involved, and its numerical solution has not yet been attempted.

In the following we discuss a metric based formulation of the "original" version of the conformal field equations, which forms the basis for Hübner's codes [10-13]. 
When deriving the conformal field equations, it turns out to be useful to start with the splitting of the Riemann tensor into its trace-free (the Weyl tensor) and trace (Ricci tensor and scalar) parts. Additionally we define the tracefree Ricci tensor $\hat{R}_{a b}=R_{a b}-g_{a b} R / 4$ and the rescaled Weyl tensor

$$
d_{a b c}{ }^{d}=\Omega^{-1} C_{a b c}{ }^{d} .
$$

The requirement that the physical scalar curvature $\tilde{R}$ vanishes implies

$$
6 \Omega \nabla^{a} \nabla_{a} \Omega=12\left(\nabla^{a} \Omega\right)\left(\nabla_{a} \Omega\right)-\Omega^{2} R,
$$

Note that this equation is not manifestly regular at $\Omega=0$, but it is actually possible to show that if (11) is satisfied at one point, then by virtue of $(12,13,14,17,18)$, to be given below, it has to be satisfied everywhere. The whole system $(11,12,13,14,17,18)$ is then regular in the sense that this point does not have to be located at $\mathscr{I}^{+}$. The vacuum Einstein equations $\tilde{R}_{a b}=0$ then yield

$$
\nabla_{a} \nabla_{b} \Omega=\frac{1}{4} g_{a b} \nabla^{c} \nabla_{c} \Omega-\frac{1}{2} \hat{R}_{a b} \Omega .
$$

Finally, commuting covariant derivatives in the expression

$$
g^{b c} \nabla_{c} \nabla_{b} \nabla_{a} \Omega
$$

and then using (12) again yields

$$
\frac{1}{4} \nabla_{a}\left(\nabla^{b} \nabla_{b} \Omega\right)=-\frac{1}{2} \hat{R}_{a b} \nabla^{b} \Omega-\frac{1}{24} \Omega \nabla_{a} R-\frac{1}{12} \nabla_{a} \Omega R .
$$

Equations for the metric can be obtained by the identity

$$
\begin{aligned}
R_{a b c}{ }^{d}= & \Omega d_{a b c}{ }^{d}+\left(g_{c a} \hat{R}_{b}{ }^{d}-g_{c b} \hat{R}_{a}{ }^{d}-g^{d}{ }_{a} \hat{R}_{b c}+g_{b}{ }_{b} \hat{R}_{a c}\right) / 2 \\
& +\left(g_{c a} g_{b}{ }^{d}-g_{c b} g_{a}{ }^{d}\right) \frac{R}{12},
\end{aligned}
$$

which defines the Weyl tensor. Expressing the Riemann tensor $R_{a b c}{ }^{d}$ in terms of the metric and its derivatives (or the Christoffel quantities in a first order formalism) yields the desired equations. Note that for the physical Riemann tensor the vacuum Einstein equations imply $\tilde{R}_{a b c}{ }^{d}=\tilde{C}_{a b c}{ }^{d}$.

We still miss differential equations for $d_{a b c}{ }^{d}$ and $\hat{R}_{a b}$. These can be obtained from the Bianchi identities $\nabla_{[a} R_{b c]} d^{e}$, which in terms of the Weyl and tracefree Ricci tensors imply

$$
\nabla_{d} \tilde{C}_{a b c}{ }^{d}=0
$$

for the Weyl tensor of a vacuum spacetime $\left(\tilde{R}_{a b}=0\right)$ and

$$
\nabla_{b} \hat{R}_{a}{ }^{b}=\frac{1}{4} \nabla_{a} R
$$

While the Weyl tensor is conformally invariant,

$$
\tilde{C}_{a b c}{ }^{d}=C_{a b c}{ }^{d},
$$


this invariance does not hold for (15). Instead however one can show that

$$
\tilde{\nabla}_{d} \tilde{C}_{a b c}{ }^{d}=\Omega \nabla_{d}\left(d_{a b c}{ }^{d}\right),
$$

which implies

$$
\nabla_{e} d_{a b c}{ }^{e}=0
$$

if the vacuum Einstein equations hold in the physical spacetime.

The Bianchi identity combined with the splitting (14) implies

$$
\nabla_{a} \hat{R}_{b c}-\nabla_{b} \hat{R}_{a c}=-\frac{1}{12}\left(\left(\nabla_{a} R\right) g_{b c}-\left(\nabla_{b} R\right) g_{a c}\right)-2\left(\nabla_{d} \Omega\right) d_{a b c}{ }^{d} .
$$

Then $(11,12,13,14,17,18)$ constitute the conformal field equations for vacuum general relativity. Here the Ricci scalar $R$ of $g_{a b}$ is considered a given function of the coordinates. For any solution $\left(g_{a b}, \hat{R}_{a b}, d_{a b c}{ }^{d}, \Omega\right), \hat{R}_{a b}$ is the traceless part of the Ricci tensor and $\Omega d_{a b c}{ }^{d}$ the Weyl tensor of $g_{a b}$. Note that the equations are regular even for $\Omega=0$.

The $3+1$ decomposition of the conformal geometry can be carried out as usual in general relativity, e.g.

$$
g_{a b}=h_{a b}-n_{a} n_{b}=\Omega^{2}\left(\tilde{h}_{a b}-\tilde{n}_{a} \tilde{n}_{b}\right),
$$

where $h_{a b}$ and $\tilde{h}_{a b}$ are the Riemannian 3-metrics induced by $g_{a b}$, respectively $\tilde{g}_{a b}$, on a spacelike hypersurface with unit normals $n_{a}$ and equivalently $n_{a}=\Omega \tilde{n}_{a}$ (our signature is $(-,+,+,+)$ ). The relation between the extrinsic curvatures $\left(\tilde{k}_{a b}=\mathcal{L}_{\tilde{n}} \tilde{h}_{a b} / 2, k_{a b}=\mathcal{L}_{n} h_{a b} / 2\right)$ is then easily derived as $k_{a b}=\Omega\left(\tilde{k}_{a b}+\Omega_{0} \tilde{h}_{a b}\right)$, where $\Omega_{0}=n^{a} \nabla_{a} \Omega$. by

The additional variables $\hat{R}_{a b}$ and $d_{a b c}^{d}$ can be decomposed into spatial objects

$$
\begin{gathered}
{ }^{(0,1)} \hat{R}_{a}=n^{b} h_{a}{ }^{c} \hat{R}_{b c}, \quad{ }^{(0,1)} \hat{R}_{a b}=h_{a}{ }^{c} h_{b}{ }^{d} \hat{R}_{c d}, \\
E_{a b}=d_{e f c d} h^{e}{ }_{a} n^{f} h^{c}{ }_{b} n^{d}, \quad B_{a b}=d_{e f c d}^{*} h^{e}{ }_{a} n^{f} h^{c}{ }_{b} n^{d},
\end{gathered}
$$

where $E_{a b}$ and $B_{a b}$ are called the electric and magnetic components of the rescaled Weyl tensor $d_{a b c}{ }^{d}$.

Note that for regular components of $h_{a b}$ and $k_{a b}$, the corresponding components of $\tilde{h}_{a b}$ and $\tilde{k}_{a b}$ with respect to the same coordinate system will in general diverge due to the compactification effect. However for the coordinate independent traces $k=h^{a b} k_{a b}, \tilde{k}=\tilde{h}^{a b} \tilde{k}_{a b}$ of the extrinsic curvatures we get

$$
\Omega k=\left(\tilde{k}+3 \Omega_{0}\right)
$$

which can be assumed regular everywhere. Note that at $\mathscr{I}, \tilde{k}=-3 \Omega_{0}$. Since $\mathscr{I}^{+}$is an ingoing null surface (with $\left(\nabla_{a} \Omega\right)\left(\nabla^{a} \Omega\right)=0$ but $\nabla_{a} \Omega \neq 0$ ), we have that $\Omega_{0}<0$ at $\mathscr{I}^{+}$. It follows that $\tilde{k}>0$ at $\mathscr{I}^{+}$. We will thus call regular spacelike hypersurfaces in $\mathcal{M}$ hyperboloidal hypersurfaces, since in $\tilde{\mathcal{M}}$ they are analogous to the standard hyperboloids $t^{2}-x^{2}-y^{2}-z^{2}=3 / \tilde{k}^{2}$ in Minkowski space, which provide the standard example. Since such hypersurfaces cross $\mathscr{I}$ 
but are everywhere spacelike in $\mathcal{M}$, they allow to access $\mathscr{I}$ and radiation quantities defined there by solving a Cauchy problem (in contrast to a characteristic initial value problem which utilizes a null surface slicing). Note that in a globally hyperbolic physical spacetime, hyperboloidal hypersurfaces will determine the future of the physical spacetime, but not all of its past, and therefore we call our studies semiglobal.

The timelike vector $t^{a}=(\partial / \partial t)^{a}$ is decomposed in the standard way into a normal and a tangential component:

$$
t^{a}=N n^{a}+N^{a}, \quad N^{a} n_{a}=0 .
$$

$N$ is called the lapse function, because it determines how fast the time evolution is pushed forward in the direction normal to $S$ and thus determines "how fast time elapses". The tangential component $N^{a}, N^{a} n_{a}=0$, shifts spatial coordinate points with time evolution, accordingly $N^{a}$ is called shift vector. The lapse $N$ and shift $N^{a}$ are not dynamical quantities, they can be specified freely and correspond to the arbitrary choice of coordinates: the lapse determines the slicing of spacetime, the choice of shift vector determines the spatial coordinates.

We will not discuss the full $3+1$ equations here for brevity, but rather refer to [10]. Their most essential feature is that they split into constraints plus symmetric hyperbolic evolution equations [10]. The evolution variables are $h_{a b}$, $k_{a b}$, the connection coefficients $\gamma^{a} b c,{ }^{(0,1)} \hat{R}_{a},{ }^{(0,1)} \hat{R}_{a b}, E_{a b}, B_{a b}$, as well as $\Omega, \Omega_{0}$, $\nabla_{a} \Omega, \nabla^{a} \nabla_{a} \Omega$ - in total this makes 57 quantities. In addition the gauge source functions $q, R$ and $N^{a}$ have to be specified. In order to guarantee symmetric hyperbolicity, they are given as functions of the coordinates. Here $q$ determines the lapse as $N=\mathrm{e}^{q} \sqrt{\operatorname{det} h}$ and $N^{a}$ is the shift vector. The Ricci scalar $R$ can be thought of as implicitly steering the conformal factor $\Omega$.

The constraints of the conformal field equations (see (14) of [10]) are regular equations on the whole conformal spacetime $\left(\mathcal{M}, g_{a b}\right)$, but they have not yet been cast into a standard type of PDE system, such as a system of elliptic PDEs (recently however, some progress in this direction has been achieved by Butscher [28]). Therefore some remarks on how to proceed in this situation are in order. A possible resolution is to resort to a 3 -step method $[11,29,30]$ :

1. Obtain data for the Einstein equations: the first and second fundamental forms $\tilde{h}_{a b}$ and $\tilde{k}_{a b}$ induced on $\tilde{\Sigma}$ by $\tilde{g}_{a b}$, corresponding in the compactified picture to $h_{a b}, k_{a b}$ and $\Omega$ and $\Omega_{0}$. This yields so-called "minimal data".

2. Complete the minimal data on $\bar{\Sigma}$ to data for all variables using the conformal constraints - in principle this is mere algebra and differentiation.

3. Extend the data from $\bar{\Sigma}$ to $\Sigma$ in some ad hoc but sufficiently smooth and "well-behaved" way.

In order to simplify the first step, numerical implementations $[11,12,30]$ so far have been restricted to a subclass of hyperboloidal slices where initially $\tilde{k}_{a b}$ is pure trace, $\tilde{k}_{a b}=\tilde{h}_{a b} \tilde{k} / 3$. The momentum constraint

$$
\tilde{\nabla}^{b} \tilde{k}_{a b}-\tilde{\nabla}_{a} \tilde{k}=0
$$


then implies $\tilde{k}=$ const. $\neq 0$. We always set $\tilde{k}>0$. In order to reduce the Hamiltonian constraint

$$
{ }^{(3)} \tilde{R}+\tilde{k}^{2}=\tilde{k}_{a b} \tilde{k}^{a b}
$$

to one elliptic equation of second order, we use a modified Lichnerowicz ansatz

$$
\tilde{h}_{a b}=\bar{\Omega}^{-2} \phi^{4} h_{a b}
$$

with two conformal factors $\bar{\Omega}$ and $\phi$. The principal idea is to choose $h_{a b}$ and $\bar{\Omega}$ and solve for $\phi$, as we will describe now. First, the "boundary defining" function $\bar{\Omega}$ is chosen to vanish on a 2 -surface $\mathcal{S}$ - the boundary of $\bar{\Sigma}$ and initial cut of $\mathscr{I}$ - with non-vanishing gradient on $\mathcal{S}$. The topology of $\mathcal{S}$ is chosen as spherical for asymptotically Minkowski spacetimes. Then we choose $h_{a b}$ to be a Riemannian metric on $\Sigma$, with the only restriction that the extrinsic 2 -curvature induced by $h_{a b}$ on $\mathcal{S}$ is pure trace, which is required as a smoothness condition [29]. With this ansatz $\tilde{h}_{a b}$ is singular at $\mathcal{S}$, indicating that $\mathcal{S}$ represents an infinity. The Hamiltonian constraint then reduces to the Yamabe equation for the conformal factor $\phi$ :

$$
4 \bar{\Omega}^{2} \Delta \phi-4 \bar{\Omega}\left(\nabla^{a} \bar{\Omega}\right)\left(\nabla_{a} \phi\right)-\left(\frac{1}{2}{ }^{(3)} R \bar{\Omega}^{2}+2 \bar{\Omega} \Delta \bar{\Omega}-3\left(\nabla^{a} \bar{\Omega}\right)\left(\nabla_{a} \bar{\Omega}\right)\right) \phi=\frac{1}{3} \tilde{k}^{2} \phi^{5} .
$$

This is a semilinear elliptic equation - except at $\mathcal{S}$, where the principal part vanishes for a regular solution. This however determines the boundary values as

$$
\phi^{4}=\frac{9}{\tilde{k}^{2}}\left(\nabla^{a} \bar{\Omega}\right)\left(\nabla_{a} \bar{\Omega}\right) .
$$

Existence and uniqueness of a positive solution to the Yamabe equation and the corresponding existence and uniqueness of regular data for the conformal field equations using the approach outlined above (assuming the "pure trace smoothness condition) have been proven by Andersson, Chruściel and Friedrich [29].

If the Yamabe equation is solved numerically, the boundary has to be chosen at $\mathcal{S}$, the initial cut of $\mathscr{I}$, with boundary values satisfying (21). If the equation were solved on a larger grid (conveniently chosen to be Cartesian), boundary conditions would have to be invented, which generically would cause the solution to lack sufficient differentiability at $\mathcal{S}$, see Hübner's discussion in [11]. This problem is due to the degeneracy of the Yamabe equation at $\mathcal{S}$. Unfortunately, this means that we have to solve an elliptic problem with spherical boundary.

The constraints needed to complete minimal initial data to data for all evolution variables split into two groups: those that require divisions by the conformal factor $\Omega$ to solve for the unknown variable, and those which do not. The latter do not cause any problems and can be solved without taking special care at $\Omega=0$. The first group, needed to compute ${ }^{(1,1)} \hat{R}, E_{a b}$ and $B_{a b}$, however does require special numerical techniques to carry out the division and furthermore it is not known whether solving them on the whole Cartesian time evolution grid actually allows solutions which are sufficiently smooth across $\mathscr{I}$. Thus, at least for these we have to find some ad-hoc extension. There are however also examples of analytically known initial data, e.g. for the Minkowski and Kruskal spacetimes, where all constraints are solved on the whole Cartesian time evolution grid. 


\section{Examples: Different Ways to Compactify Minkowski Spacetime}

The examples presented in this section help to illustrate the compactification procedure - in particular its inherent gauge freedom. They yield interesting numerical tests, some of which will be presented in Sect. 5 .

\subsection{Almost Static Compactification of Minkowski Spacetime}

From the perspective of hyperboloidal initial data, the simplest way to compactify Minkowski spacetime is to choose the initial conformal three-metric as the flat metric, $h_{a b}=\delta_{a b}$, to set $k_{a b}=h_{a b}$, which solves the momentum constraint (20) and to choose the conformal curvature scalar $R_{g}{ }^{3}$ as spherically symmetric, $R_{g}=R_{g}\left(x^{2}+y^{2}+z^{2}\right)$. We know from [29] that a unique solution to the constraints exists. It is not hard to see that it has to be spherically symmetric. Furthermore, it is topologically trivial. From Birkhoff's theorem we can thus conclude that we are dealing with Minkowski spacetime. Choosing the simplest gauge $q=0, N^{a}=0, R_{g}=0$, the resulting unphysical spacetime is actually Minkowski spacetime in standard coordinates:

$$
\mathrm{d} s^{2}=-\mathrm{d} t+\mathrm{d} \Sigma^{2}=\Omega^{2}\left(-\mathrm{d} T^{2}+\mathrm{d} R^{2}+R^{2}\left(\mathrm{~d} \theta^{2}+\sin ^{2} \theta \mathrm{d} \phi^{2}\right)\right),
$$

where $\mathrm{d} \Sigma^{2}$ is the standard metric on $\mathbb{R}^{3}, \mathrm{~d} \Sigma^{2}=\mathrm{d} r^{2}+r^{2}\left(\mathrm{~d} \theta^{2}+\sin ^{2} \theta \mathrm{d} \phi^{2}\right)$, and the conformal factor is

$$
\Omega=\left(R^{2}-T^{2}\right)^{-1}=\left(r^{2}-t^{2}\right)
$$

where

$$
r=\frac{R}{R^{2}-T^{2}}, \quad t=\frac{T}{R^{2}-T^{2}} .
$$

This setup has been chosen as the basis of Hübner's numerical study of weak data evolutions [13]. With the initial cut of $\mathscr{I}^{+}$chosen at $x^{2}+y^{2}+z^{2}=1, i^{+}$ is located at coordinate time $t=1$, the generators of $\mathscr{I}^{+}$being straight lines at an angle of $45^{\circ}$.

This conformal representation of Minkowski spacetime is an "almost static" gauge - since the spatial geometry is time-independent, so are all evolution variables except for the conformal factor $\Omega$. The physical region inside of $\mathscr{I}^{+}$contracts to the regular point $i^{+}$within finite time. This feature is shared with the standard "textbook" example of conformally compactifying Minkowski spacetime, which takes the form of a map into part of the Einstein static universe with $R_{g}=6$,

$$
\mathrm{d} s^{2}=-\mathrm{d} t^{2}+\mathrm{d} \Sigma^{2}=\Omega^{2}\left(-\mathrm{d} T^{2}+\mathrm{d} R^{2}+R^{2}\left(\mathrm{~d} \theta^{2}+\sin ^{2} \theta \mathrm{d} \phi^{2}\right)\right),
$$

\footnotetext{
${ }^{3}$ We change notation from $R$ to $R_{g}$ for this section to avoid confusion with a coordinate $R$ we will introduce below.
} 
where $\mathrm{d} \Sigma^{2}$ is the standard metric on $S^{3}, \mathrm{~d} \Sigma^{2}=\mathrm{d} \varrho^{2}+\sin ^{2} \varrho\left(\mathrm{d} \theta^{2}+\sin ^{2} \theta \mathrm{d} \phi^{2}\right)$, and the conformal factor is

$$
\Omega^{2}=4\left(1+(T-R)^{2}\right)^{-1}\left(1+(T+R)^{2}\right)^{-1}=4 \cos ^{2} \frac{t-\varrho}{2} \cos ^{2} \frac{t+\varrho}{2} .
$$

Here the coordinate transformations are

$$
\begin{aligned}
& \varrho=\arctan (T+R)-\arctan (T-R), \\
& t=\arctan (T+R)+\arctan (T-R) .
\end{aligned}
$$

In these coordinates Minkowski spacetime corresponds to the coordinate ranges

$$
\begin{gathered}
-\pi<t+\varrho<\pi, \\
-\pi<t-\varrho<\pi, \\
\varrho \geq 0 .
\end{gathered}
$$

For details and pictures of this mapping see the discussions by [4], [15] or [16].

Alternatively, we can choose stereographic spatial coordinates such that

$$
\mathrm{d} \Sigma^{2}=\omega^{2}\left(\mathrm{~d} r^{2}+r^{2}\left(\mathrm{~d} \theta^{2}+\sin ^{2} \theta \mathrm{d} \phi^{2}\right)\right), \quad \omega=\frac{2}{\left(1+r^{2}\right)} .
$$

or we may absorb the spatial conformal factor into the spacetime conformal factor by rescaling to

$$
\mathrm{d} s^{\prime 2}=-\omega^{-2} \mathrm{~d} t^{2}+\mathrm{d} r^{2}+r^{2}\left(\mathrm{~d} \theta^{2}+\sin ^{2} \theta \mathrm{d} \phi^{2}\right),
$$

which yields the lapse to be $N=1(q=-3 \log \omega)$, respectively $N=\omega^{-2}$ $(q=-2 \log \omega)$. Note that in the numerical code we use Cartesian coordinates $x=r \sin \theta \cos \phi, y=r \sin \theta \sin \phi, z=r \cos \theta$.

The conformal transformation leading to (29) changes the scalar curvature from $R_{g}=6$ to $R_{g}=-12\left(1+r^{2}\right)^{-2}$. We will see below in Sect. 5 that these simple variations in gauge source functions and conformal rescaling lead to numerical representations which are quite different, e.g. with regard to accuracy and robustness.

\subsection{A Static Hyperboloidal Gauge for Minkowski Spacetime}

By translating a standard hyperboloid in Minkowski spacetime along the trajectories of the $\partial / \partial t$ Killing vector, one can obtain a gauge where not only the conformal spacetime is static, but also the conformal factor is time-independent - thus also the physical geometry and all evolution variables of the conformal field equations can be made time independent (this has been pointed out to me by M. Weaver and I essentially follow her notes below). See also a talk given by V. Moncrief [31], which we have become aware of after starting to work with this gauge. 
In this gauge the point $i^{+}$is not brought into a finite distance and remains in the infinite future. This conformal gauge is particularly useful for stability tests.

To derive this static metric, we start with spherical coordinates $(T, R, \theta, \phi)$ on Minkowski space, where the metric is

$$
\mathrm{d} \tilde{s}^{2}=-\mathrm{d} T^{2}+\mathrm{d} R^{2}+R^{2}\left(\mathrm{~d} \theta^{2}+\sin ^{2} \theta \mathrm{d} \phi^{2}\right) .
$$

A family of standard hyperboloids with time translation parameter $t$ is given by

$$
(T-t)^{2}-R^{2}=1
$$

We transform now to new coordinates $(t, \varrho, \theta, \phi)$, where the level surfaces of $t$ are the standard hyperboloids and $\varrho(R)$ is chosen as a new radial parameter on the hyperboloids. Setting $T=t+\cosh \varrho$ and $R=\sinh \varrho$, the physical metric becomes

$$
\mathrm{d} \tilde{s}^{2}=-\mathrm{d} t^{2}-2 \sinh \varrho \mathrm{d} \varrho \mathrm{d} t+\mathrm{d} \varrho^{2}+\sinh ^{2} \varrho\left(\mathrm{d} \theta^{2}+\sin ^{2} \theta \mathrm{d} \phi^{2}\right) .
$$

For simplicity we choose the conformal three-metric to be flat and introduce new spherical coordinates $(r, \theta, \phi)$ such that

$$
\left.\mathrm{d} s^{2}\right|_{t=\text { const. }}=\mathrm{d} r^{2}+r^{2}\left(\mathrm{~d} \theta^{2}+\sin ^{2} \theta \mathrm{d} \phi^{2}\right) .
$$

Since $h_{a b}=\Omega^{2} \tilde{h}_{a b}$ we get $\Omega=\mathrm{d} r / \mathrm{d} \varrho$ and

$$
\int_{\varrho}^{\infty} \frac{\mathrm{d} \varrho^{\prime}}{\sinh \varrho}=\int_{r}^{1} \frac{\mathrm{d} r^{\prime}}{r^{\prime}} .
$$

The limits of integration are given by the fact that $\lim _{\varrho \rightarrow \infty} r=1$. Performing the integrals one finds that

$$
r=\frac{\mathrm{e}^{\varrho}-1}{\mathrm{e}^{\varrho}+1}=\frac{1}{R}\left(\sqrt{1+R^{2}}-1\right)
$$

and

$$
\Omega=\frac{1-r^{2}}{2},
$$

our choice thus maps $\mathscr{I}^{+}$to the timelike cylinder $r=1$.

The computer time coordinate $t$ is a Bondi time coordinate on $\mathscr{I}$. In coordinates $(t, r, \theta, \phi)$, the conformal metric reads

$$
\mathrm{d} s^{2}=-\Omega^{2} \mathrm{~d} t^{2}-2 r \mathrm{~d} r \mathrm{~d} t+\mathrm{d} r^{2}+r^{2}\left(\mathrm{~d} \theta^{2}+\sin ^{2} \theta \mathrm{d} \phi^{2}\right),
$$

or

$$
\mathrm{d} s^{2}=-\Omega^{2} \mathrm{~d} t^{2}-2 \mathrm{~d} t(x \mathrm{~d} x+y \mathrm{~d} y+z \mathrm{~d} z)+\mathrm{d} x^{2}+\mathrm{d} y^{2}+\mathrm{d} z^{2}
$$

in Cartesian coordinates, $x=r \sin \theta \cos \phi, y=r \sin \theta \sin \phi, z=r \cos \theta$, which are used in the numerical code. The shift vector is thus given by $N^{i}=-x^{i}$ and the lapse can be computed from $-N^{2}+h_{a b} N^{a} N^{b}=g_{t t}$ (as implied by (19)) as 
$N=\left(1+r^{2}\right) / 2$. The three metric has unit determinant, so $q=\ln N$. Note that the shift vector does not become "superluminal" beyond $\mathscr{I}^{+}$, because the lapse is growing faster than the shift; $g_{t t}$ is nonnegative everywhere and zero only at $\mathscr{I}^{+}$. The conformal Ricci scalar is

$$
R_{g}=12 \frac{\left(1-r^{2}\right)\left(3+r^{2}\right)}{\left(1+r^{2}\right)^{3}},
$$

which vanishes at $\mathscr{I}^{+}$.

For a numerical calculation one needs the minimal initial data set,

$$
\left(h_{a b}, \Omega, k_{a b}, \Omega_{0}\right)
$$

and the gauge source functions, $\left(R, N, N^{a}\right)$. In a numerical calculation in which the Yamabe equation is solved to find $\Omega$, one gives $\left(h_{a b}, \bar{\Omega}, \operatorname{tr} k\right)$. In a test case such as this, which is an explicitly known solution, one can just take $\bar{\Omega}=\Omega$. It remains therefore to calculate $k_{a b}$ and $\Omega_{0}$. From

$$
k_{a b}=\frac{1}{2 N}\left(\partial_{t} h_{a b}-\mathcal{L}_{N} h_{a b}\right)
$$

we find that the components of the extrinsic curvature are $k_{i j}=\delta_{i j} / N$ and $k=3 / N$. From the identity $\tilde{k}=\Omega k-3 \Omega_{0}$ we find that

$$
\Omega_{0}=-\frac{2 r^{2}}{1+r^{2}} .
$$

\section{History}

This section tries to give a broad overview of what has been achieved so far in the field of numerical treatment of the conformal field equations. Historically, this field was started by Peter Hübner by studying a scalar field coupled to gravity in spherical symmetry in his $\mathrm{PhD}$ thesis [32] finished in 1993. His subsequent work has lead to the development of both a $2 \mathrm{D}$ and a $3 \mathrm{D}$ evolution code, formulated in "metric" variables. Jörg Frauendiener has also developed an independent 2D code, formulated in frame variables.

\subsection{Early Work on Spherical Symmetry}

The first numerical implementation of the conformal field equations is due to Peter Hübner, who has studied the spherically symmetric collapse of scalar fields in his $\mathrm{PhD}$ thesis [32] and subsequently in [33]. In his gauge both future null infinity $\left(\mathscr{I}^{+}\right)$and future timelike infinity $\left(i^{+}\right)$are compactified and the whole spacetime is covered in finite coordinate time. Hübner studies the global structure of the spacetime, including the appearance of singularities and the localization of the event horizon. To handle the latter, floating point exceptions are caught and grid points are flagged as "singular", grid points whose values depend on information 
from singular grid points are correspondingly flagged as singular as well. Even though this method does not allow to actually trace the singularity in a strict sense (computers cannot actually deal with infinite values), the method traces the singularity as tightly as possible. In contrast to typical black hole excision schemes, which are based on locating the apparent horizon, this scheme could thus be termed "tight excision". The method has not yet been implemented in higher dimensions, where one has to face more intricate technical problems and where the structure of the singularity is likely to be much more complex as well. The paper also studies critical collapse. Hübner's results are consistent with the black hole mass power-law scaling with the correct exponent, however no echoing related to discrete self-similarity has been seen in his results. This has created some discussion, whether the results of other authors are numerical artefacts, or artefacts of boundary conditions at finite distance. However numerical critical collapse simulations in a compactified characteristic framework have recently shown both the correct power-law scaling and discretely self-similar echoing [34].

The coordinates in this approach are based on the geometric structure of double null-coordinates that is available in spherical symmetry. Unfortunately this choice does not generalize in the absence of spherical symmetry. Finding a gauge that would allow to run, say, the Kruskal spacetime in a 3D code for "arbitrarily long" Bondi times is an open problem, where significant insight could be gained from studying more general gauges in a manifestly spherically symmetric code.

\subsection{Axially Symmetric Spacetimes with Toroidal $\mathscr{I}$ in the Frame Formulation}

Following Hübner's encouraging results for spherically symmetric simulations $[32,33]$, numerical codes have been developed by Frauendiener and Hübner to study axially symmetric spacetimes. For simplicity, e.g. to avoid numerical stability problems at the axis of symmetry and to avoid problems associated with a $\mathscr{I}$ of spherical topology - which does not align with Cartesian coordinates - both Hübner and Frauendiener considered the asymptotically A3-spacetimes [35,36], which do not possess an axis of symmetry and where $\mathscr{I}$ has toroidal topology. These spacetimes are modelled after the A3-metric in the Ehlers-Kundt classification [37], which provides an analogue of the Schwarzschild metric in plane symmetry. These spacetimes are not physical, but they contain a large class of nontrivial radiative vacuum spacetimes, which make them an interesting toy model to study numerical techniques, gauges and the extraction of radiation. ${ }^{4}$ These axisymmetric codes thus have been designed to treat the vacuum case, and matter couplings have not yet been implemented. An advantage for code-testing is that exact solutions are known $[35,38]$.

In the first [27] of a series of papers [24,26,27,30] on his axisymmetric code, Frauendiener gives a nice overview of the motivation for using the conformal

\footnotetext{
${ }^{4}$ One of the notable differences with the Minkowski case is that one can only define a Bondi-mass but no Bondi four-momentum.
} 
field equations of numerical simulations of isolated systems. He discusses the conformal field equations in the space spinor formalism [39], which is chosen because of compactness of notation, and because it allows a very straightforward $3+1$ decomposition of the equations, rendering the equations in symmetric hyperbolic form. His formalism contains 8 free functions which determine the gauge: the harmonicity $F:=\nabla_{c} \nabla^{c} t$ determines the choice of time coordinate $t$, the shift is given in terms of frame coefficients, the scalar curvature $R$ ( $\Lambda$ in his notation) of the compactified spacetime and an imaginary and symmetric space spinor field $F_{A B}$ (i.e. three numbers), which determines rotations of the spatial frame (for $F_{A B}=0$ the frame is transported via Fermi-Walker transport). $\mathrm{He}$ also discusses the implications of the assumptions of the toroidal symmetry, in particular for the choice of gauge - e.g. the adoption of the frame.

In the second paper [26] of the series, Frauendiener discusses his numerical methods and gauge choices and presents results for evolutions of initial data corresponding to the exact solution presented in [35]. Here one of the two Killing vectors is disguised by a coordinate transformation. The numerical evolution proceeds via a generalization of the Lax-Wendroff scheme to 2D, which Frauendiener proves to be stable and second order accurate. The time step is such that the numerical domain of dependence is contained in the domain of dependence as defined by the equations. An essential difficulty - as usual - is posed by the treatment of the boundary. Well-posedness of the associated initial-boundaryvalue problem has not yet been proven and numerical analysis can only provide rough guidelines to work out stable algorithms [40]. Frauendiener's boundary treatment is based on the identification of ingoing and outgoing modes at the boundary, as determined from the symmetric hyperbolic character of the equations. He sets boundary values for inward-propagating quantities (e.g. motivated by the exact solution) and sets values for the outward propagating quantities by extrapolation from the interior. This method can be applied just a few grid-points outside of $\mathscr{I}$ and is found to be stable as long as the gauge source functions do not depend on the evolution variables - which would change the characteristics. Note that the constraints will in general not be satisfied on the boundary, which may trigger constraint-violating modes of the equations.

Frauendiener gives a detailed discussion of the problems associated with the choice of the gauge and performs a number of numerical experiments in this respect, evolving data corresponding to exact solutions [35,38] with singular $i^{+}$. One of the problems is that if the gauge source functions are allowed to depend on the evolution variables, this will change the characteristics of the system and will in general spoil the symmetric hyperbolic character of the system. Experiments in this direction, where $F=F(N, K)$, indeed exhibited a boundary instability. Regarding the choice of time coordinate, that is, the harmonicity function $F$, several choices are tested: a "natural" gauge, which is taken from the exact solution, the Gauss gauge (where the lapse $N$ is spatially constant), the harmonic gauge, $F=0$ and a family of gauges that interpolates between the "natural" and harmonic gauge. 
The "natural" gauge is found to provide the best performance and the approach to the singularity is found to be essentially limited by machine precision. The harmonic gauge leads to a coordinate singularity before reaching the singularity. This feature is shared by most of the gauges that interpolate between natural and harmonic gauge. For the "Gauss" gauge with $N=$ const., caustics (coordinate shocks) develop quickly and crash the simulations.

Regarding the choice of shift vector, a prescription for $\mathscr{I}$ fixing - that is, steering the evolution of the surface $\Omega=0$ - is discussed, which can be easily implemented in Frauendiener's formulation. This however relies on the specific form of the frame equations and does not carry over to equations as those used in Hübner's codes [10]. In particular he studies the case of " $\mathscr{I}$ freezing" - holding the coordinate position of $\mathscr{I}$ in place such that no loss of resolution occurs in the physical domain.

Finally, he discusses the extraction of gravitational radiation, e.g. by computing the Bondi mass and shows some results.

In order to study more general spacetimes, Frauendiener has implemented a numerical scheme for determining hyperboloidal initial data sets for the conformal field equations by using pseudo-spectral methods as described in [30]. He uses the implicit approach of first solving the Yamabe equation and then carrying out the division by the conformal factor for certain fields which vanish on $\mathscr{I}$. The challenge there is to numerically obtain a smooth quotient. The division problem is treated by a transformation to the coefficient space, where a QR-factorization of a suitable matrix is used and then transforming back.

In [24] Frauendiener gives a pedagogical discussion of the issue of radiation extraction in asymptotically flat space-times within the framework of conformal methods for numerical relativity. The aim is to show that there exists a well defined and accurate extraction procedure which mimics the physical measurement process and operates entirely intrinsically within $\mathscr{I}^{+}$. The notion of a detector at infinity is defined by idealizing local observers in Minkowski space. A detailed discussion is presented for Maxwell fields and the generalization to linearized and full gravity is performed by way of the similar structure of the asymptotic fields.

Recently, Hein has written a 2D axisymmetric code that allows for an axis [41], i.e. can treat the physical situation with a $\mathscr{I}^{+}$of spherical topology. The usual problem of the coordinate singularity at the axis in adapted coordinates is solved by using Cartesian coordinates, following a method developed by Alcubierre et al. [42]. The code has so far been tested by evolving Minkowski spacetime in various gauges, further tests with nontrivial spacetimes are currently underway.

\subsection{Metric-Based 2D and 3D Codes}

The basic design of Hübner's approach is outlined in [10], where he presents the first order time evolution equations as obtained from a $3+1$ split of the conformal field equations. The evolution equations can be brought into symmetric 
hyperbolic form by a change of variables. He discusses his motivation of avoiding artificial boundaries and how the conformal field equations formally allow placement of the grid boundaries outside the physical spacetime.

A particularly subtle part of the evolution is usually the boundary treatment. In the conformal approach we are in the situation that the boundary can actually be placed outside of the physical region of the grid - this is one of its essential advantages! In typical explicit time evolution algorithms, such as our RungeKutta method of lines, the numerical propagation speed is larger than the speed of all the characteristics (in our case the speed of light). Thus $\mathscr{I}$ does not shield the physical region from the influence of the boundary - but this influence has to converge to zero with the convergence order of the algorithm - fourth order in our case. In principle one therefore does not have to choose a "physical" boundary condition. The only requirements are stability and "practicality" - e.g. the boundary condition should avoid, if possible, the development of large gradients in the unphysical region to reduce the numerical "spill over" into the physical region, or even code crashes. It seems likely however, that this practicality requirement will eventually lead to a treatment of the boundary which satisfies the constraints at the boundary.

Hübner develops the idea of modifying the equations near the grid boundaries to obtain a consistent and stable discretization. The current implementation of the boundary treatment relies on this introduction of a "transition layer" in the unphysical region, which is used to transform the rescaled Einstein equations to trivial evolution equations, which are stable with a trivial copy operation at outermost gridpoint as a boundary condition (see [10] for details and references). He thus replaces

$$
\partial_{t} f+A^{i} \partial_{i} f-b=0
$$

by

$$
\partial_{t} f+\alpha(\Omega)\left(A^{i} \partial_{i} f-b\right)=0,
$$

where $\alpha$ is chosen as $\alpha(\Omega)=0$ for $\Omega \leq \Omega_{0}<\Omega_{1}<0$ and 1 for $\Omega \geq \Omega_{1}$. One potential problem is that the region of large constraint violations outside of $\mathscr{I}$ may trigger constraint violating modes of the equations that can grow exponentially. Another problem is that a "thin" transition zone causes large gradients in the coefficients of the equations - thus eventually leading to large gradients in the solution, while a "thick" transition zone means loosing many gridpoints. If no transition zone is used at all and the Cartesian grid boundary touches $\mathscr{I}$, the ratio of the number of grid points in the physical region versus the number of grid points in the physical region is already $\pi / 6 \approx 0.52$.

Furthermore he discusses his point of view concerning possible advantages of the conformal approach and discusses potential problems of the Cauchy and Cauchy-Characteristic matching approaches to numerical relativity. He outlines the geometric scenario of his approach and stresses that these techniques allow, in principle, to calculate the complete future of scenarios such as initial data for $N$ black holes.

The second paper [11] of the series deals with the technical details of construction of initial data and of time-evolution of such data. The second and 
fourth order discretizations, which are used for the construction of the complete data set and for the numerical integration of the time evolution equations, are described and their efficiencies compared. Results from tests for $A 3$ and disguised Minkowski spacetimes confirm convergence for the 2D and 3D codes.

The simplest approach to the division by $\Omega$ would be implementing l'Hôpital's rule. However this leads to non-smooth errors and consequently to a loss of convergence [11]. Instead, Hübner [11] has developed a technique to replace a division $g=f / \Omega$ by solving an elliptic equation of the type

$$
\nabla^{a} \nabla_{a}\left(\Omega^{2} g-\Omega f\right)=0
$$

for $g$ (actually some additional terms added for technical reasons are omitted here for simplicity). For the boundary values $\Omega^{2} g-\Omega f=0$, the unique solution is $g=f / \Omega$. The resulting linear elliptic equations for $g$ are solved by the same numerical techniques as the Yamabe equation. For technical details see Hübner $[12]$.

Finally, we have to extend the initial data to the full Cartesian spatial grid in some way. Since solving all constraints also outside of $\mathscr{I}$ will in general not be possible in a sufficiently smooth way [11], we have to find an ad hoc extension, which violates the constraints outside of $\mathscr{I}$ but is sufficiently well behaved to serve as initial data. The resulting constraint violation is not necessarily harmful for the evolution, since $\mathscr{I}$ causally disconnects the physical region from the region of constraint violation. On the numerical level, errors from the constraint violating region will in general propagate into the physical region, but if our scheme is consistent, these errors have to converge to zero with the convergence order of the numerical scheme (fourth order in our case). There may of course still be practical problems that prevent us from reaching this aim: making the ad-hoc extension well behaved is actually quite difficult, the initial constraint violation may trigger constraint violating modes in the equations, which take us away from the true solution, singularities may form in the unphysical region, etc.

Since the time evolution grid is Cartesian, its grid points will in general not coincide with the collocation points of the pseudo-spectral grid. Thus fast Fourier transformations cannot be used for transformation to the time evolution grid. The current implementation instead uses standard discrete ("slow") Fourier transformations, which typically take up the major part of the computational effort of producing initial data.

It turns out that the combined procedure works reasonably well for certain data sets. For other data sets the division by $\Omega$ is not yet solved in a satisfactory way and constraint violations are of order unity for the highest available resolutions. In particular this concerns the constraint $\nabla_{b} E_{a}{ }^{b}=-{ }^{(3)} \varepsilon_{a b c} k^{b d} B_{d}{ }^{c}$ $((14 \mathrm{~d})$ in $[10])$, since $E_{a b}$ is computed last in the hierarchy of variables and requires two divisions by $\Omega$. Further research is required to analyze the problems and either improve the current implementation or apply alternative algorithms. Ultimately, it seems desirable to change the algorithm for obtaining initial data to a method that solves the conformal constraints directly and therefore does 
not suffer the current problems. This approach may of course introduce new problems like having an elliptic system too large to be handled in practice.

The time evolution algorithm is an implementation of a standard fourth order method of lines (see e.g. [43]). In the method of lines we formally write

$$
\partial_{t} f=B\left(f, \partial_{i} f\right)
$$

where $B\left(f, \partial_{i} f\right)=-A^{i}(f) \partial_{i} f+b(f)$. Discretizing the spatial derivatives parametrizes the ordinary differential equations by grid point index. For the present code, fourth order accurate centered spatial differences have been implemented, e.g. for the $x$-derivative:

$$
\partial_{x} f \rightarrow \frac{1}{12 \Delta x}\left(-f_{i+2, j, k}+8 f_{i+1, j, k}-8 f_{i-1, j, k}+f_{i-1, j, k}\right) .
$$

The numerical integration of the ordinary differential equations proceeds via the standard fourth order Runge-Kutta scheme:

$$
f_{i, j, k}^{l+1}=f_{i, j, k}^{l}+\frac{1}{6}\left(k_{i, j, k}^{l}+2 k_{i, j, k}^{l+1 / 4}+2 k_{i, j, k}^{l+1 / 2}+k_{i, j, k}^{l+3 / 4}\right),
$$

where

$$
\begin{array}{ll}
k_{i, j, k}^{l}=\Delta t B\left(f_{i, j, k}^{l}, \partial_{i} f_{i, j, k}^{l}\right), & \\
k_{i, j, k}^{l+1 / 4}=\Delta t B\left(f_{i, j, k}^{l+1 / 4}, \partial_{i} f_{i, j, k}^{l+1 / 4}\right), & f_{i, j, k}^{l+1 / 4}=f_{i, j, k}^{l}+\frac{1}{2} k_{i, j, k}^{l}, \\
k_{i, j, k}^{l+1 / 2}=\Delta t B\left(f_{i, j, k}^{l+1 / 2}, \partial_{i} f_{i, j, k}^{l+1 / 2}\right), & f_{i, j, k}^{l+1 / 2}=f_{i, j, k}^{l}+\frac{1}{2} k_{i, j, k}^{l+1 / 4}, \\
k_{i, j, k}^{l+3 / 4}=\Delta t B\left(f_{i, j, k}^{l+3 / 4}, \partial_{i} f_{i, j, k}^{l+3 / 4}\right), & f_{i, j, k}^{l+3 / 4}=f_{i, j, k}^{l}+k_{i, j, k}^{l+1 / 2} .
\end{array}
$$

Additionally, a dissipation term of the type discussed in theorems 6.7 .1 and 6.7.2 of Gustafsson, Kreiss and Oliger [43] is added to the right-hand-sides to damp out high frequency oscillations and keep the code numerically stable. The dissipation term used is

$$
\sigma Q_{2}:=\frac{\sigma}{64 N}(\Delta x)^{5} \sum_{i=1}^{N} \partial_{i}{ }^{6} f
$$

where the spatial derivatives are discretized as

$$
\begin{gathered}
\partial_{x}{ }^{6} f_{i, j, k}^{l} \rightarrow \frac{1}{(\Delta x)^{6}}\left(f_{i-3, j, k}^{l}-6 f_{i-2, j, k}^{l}+15 f_{i-1, j, k}^{l}\right. \\
\left.-20 f_{i, j, k}^{l}+15 f_{i+1, j, k}^{l}-6 f_{i+2, j, k}^{l}+f_{i+3, j, k}^{l}\right) .
\end{gathered}
$$

Numerical experiments show that usually small amounts of dissipation ( $\sigma$ of the order of unity or smaller) are sufficient and do not change the results in any significant manner. Numerical tests for Minkowski spacetime with disguised 
symmetries and an explicitly known A3-like solution with radiation [38] are described in [11].

Further extensive tests of the 2D code have been performed by Weaver [44]. She studied the choice of gauge source functions for an A3-like solution, solving the Yamabe equation for the conformal factor. She found that for this solution it is quite simple to prescribe a shift so that $\mathscr{I}$ is fixed to a very good approximation. She also studied the use of the gauge source function $q$ to prolong the numerical simulation inside physical spacetime. In cases where $q=0$ results in a "singularity" developing outside physical spacetime (which causes the code to crash), prescription of $q$ so that the evolution inside physical spacetime is prolonged compared to the outside allows the simulation to essentially cover the physical spacetime to the future of the initial data surface. She thus found that in this context the ad hoc prescription of gauge source functions was sufficient to achieve desired effects, and caused no instabilities. Also she explored the effect of turning off the transition zone, while still simply copying data at the outer grid boundary into the ghost zone, along with prescription of $q$, so that the evolution is slowed down at the outer boundary. In the A3-like 2D runs this alternative boundary treatment was successful and avoided problems created by the transition zone.

In the third part of the series [12], a pseudospectral solver for the constraints is described. Since the implementation depends on the topology, it discusses both the asymptotically A3 and asymptotically Minkowski cases. At the end also some remarks are made about a possible extension to the multi-blackhole case, using a multi-patch scheme (the Schwarz alternating procedure).

In the fourth part of the series [13] Hübner presents results of 3D calculations for initial data which evolve into a regular point $i^{+}$and which thus could be called "weak data". The initial conformal metric is chosen in Cartesian coordinates as

$$
\mathrm{d} s^{2}=\left(1+\frac{A}{3} \bar{\Omega}^{2}\left(x^{2}+2 y^{2}\right)\right) \mathrm{d} x^{2}+\mathrm{d} y^{2}+\mathrm{d} z^{2} .
$$

We choose $\bar{\Omega}=\left(1-\left(x^{2}+y^{2}+z^{2}\right)\right) / 2$ as the boundary defining function $\bar{\Omega}$ appearing in this ansatz. It is used to satisfy the smoothness condition for the conformal metric at $\mathscr{I}$. For the gauge source functions, Hübner has made the "trivial" choice: $R=0, N^{a}=0, q=0$, i.e. the conformal spacetime has vanishing scalar curvature, the shift vanishes and the lapse is given by $N=\mathrm{e}^{q} \sqrt{\operatorname{det} h}=$ $\sqrt{\operatorname{det} h}$. This simplest choice of gauge is completely sufficient for $A=1$ data and has lead to a milestone result of the conformal approach - the evolution of weak data which evolve into a regular point $i^{+}$of $\mathcal{M}$, which is resolved as a single grid cell. With this result Hübner has illustrated a theorem by Friedrich, who has shown that for sufficiently weak initial data there exists a regular point $i^{+}$of $\mathcal{M}$ [45]. The complete future of (the physical part of) the initial slice can thus be reconstructed in a finite number of computational time steps. This calculation is an example of a situation for which the usage of the conformal field equations is ideally suited: main difficulties of the problem are directly addressed and solved by using the conformal field equations. 
A natural next question to ask is: what happens if one increases the amplitude $A$ ? To answer this question, I have performed and analyzed runs for integer values of $A$ up to $A=20$. Preliminary results have been presented in [46]. While for $A=1,2$ the code was found to be able to continue beyond $i^{+}$without problems, for all higher amplitudes the "trivial" gauge leads to code crashes before reaching $i^{+}$. While the physical data still decay quickly in time, a sharp peak of the lapse develops outside of $\mathscr{I}$ and crashes the code after Bondi time $\sim 8(320 M)$ for $A=3$ and $\sim 1.5(3 M)$ for $A=20$ (here $M$ is the initial Bondi mass). A partial cure of the problem was obtained using a modified gauge source function $q=-r^{2} / a\left(N=\mathrm{e}^{-r^{2} / a} \sqrt{\operatorname{det} h}\right)$, where $a$ is tuned such that one gets a smooth lapse and smooth metric components. For $A=5$, for example, a value of $a=1$ was found by moderate tuning of $a$ (significantly decreasing or increasing $a$ crashes the code before the regular $i^{+}$is reached). Unfortunately, this modification of the lapse is not sufficient to achieve much higher amplitudes. As $A$ is increased, the parameter $a$ requires more fine tuning, which was only achieved for $A \leq 8$. For higher amplitudes the code crashes with significant differences in the maximal and minimal Bondi time achieved, while the radiation still decays very rapidly. Furthermore, the curvature quantities do not show excessive growth - it is thus natural to assume that we are still in the weakfield regime and the crash is not connected to the formation of an apparent horizon or singularity. While some improvement is obviously possible through simple non-trivial models for the lapse (or other gauge source functions), this approach seems quite limited and more understanding will be necessary to find practicable gauges. An interesting line of research would be to follow the lines of [47] in order to find evolution equations for the gauge source functions which avoid the development of pathologies.

Schmidt has presented hyperboloidal initial data for the Kruskal spacetime, a hyperboloidal foliation for the future of these hyperboloidal initial data [48] and results from numerical simulations evolving these initial data with different gauges, which have been performed by Weaver with Hübner's 3D code. The explicit hyperboloidal version of the Kruskal spacetime is very useful for numerically testing the conformal approach in the treatment of black hole spacetimes. These runs have been performed in octant mode. The runs typically proceed until the determinant of the three metric becomes negative [44], caused by some feature in the exact solution which is no longer adequately resolved and which is growing, leading to large narrow spikes in the numerical data. Future work will have to be directed toward improving the choice of gauge source functions such that rapidly growing sharp features are avoided.

In the next section, I will present new results obtained with the 3D code for asymptotically Minkowski spacetimes, which will illustrate some of the current problems. One of these is the presence of exponentially growing constraint violating modes. The problem of controlling the growth of the constraints for the conformal field equations has first been addressed by Florian Siebel in a diploma thesis [49] and subsequently by Hübner and Siebel in [50]. The key idea in this work is to develop a $\lambda$-system [51] for the conformal field equations in $1+1$ 
dimensions (with toroidal $\mathscr{I}$ 's). A $\lambda$-system is an enlarged evolution system, where evolution equations for the constraints are added in, consistently with symmetric hyperbolicity. One then has a large parameter space of coefficient functions available in which to find choices such that the new system has the constraint surface as an attractor. The main conclusion of this work is that it was not possible to significantly improve the fidelity of the numerical calculations. In those cases where moderate improvements regarding the constraints could be achieved, the deviation from the known exact solution would get larger.

\section{$5 \quad$ Results from 3D Calculations}

All the results presented in this section have been performed with $121^{3}$ grids on 32 processors of the AEI's SGI origin 2000. The outer boundary has been placed at a radius of $r=1.15$ in these runs $\left(\mathscr{I}^{+}\right.$is initially located at a radius $\left.r=1\right)$.

\subsection{Minkowski Data}

We will first discuss some results for Minkowski spacetime, which in spite of its simplicity provides some nontrivial numerical tests. As has been first demonstrated by Hübner in [13], for weak data - in particular Minkowski space - it is possible via the conformal approach to cover the whole domain of dependence of initial data reaching out to $\mathscr{I}^{+}$with a finite number of time steps. Let us thus first consider the gauges of Sect. 3.1, where the compactified geometry is time-independent, but a time-dependent conformal factor $\Omega$ is responsible for contracting the cuts of $\mathscr{I}^{+}$to a point within finite coordinate time.

We have compared the gauges where the conformal spacetime is Minkowski, (22), the Einstein static universe (23), or the spacetime given by (29). Essentially, the result is that the Minkowski case yields the highest accuracy, the Einstein universe case works in principle and in the case (29) the code crashes before reaching $i^{+}$. In Fig. 1 the Minkowski and Einstein universe cases are compared by plotting $h_{x x}-1$ and the value of the constraint $\nabla_{x} \Omega=\Omega_{x}$ at the center versus coordinate time (where $t$ is scaled such that $t\left(i^{+}\right)=1$. The Minkowski case - denoted by the unbroken line - clearly yields better accuracy, although the growth of $h_{x x}-1$ is faster and approximately exponential during the later stage of "physical" evolution. Note that the constraint grows very fast in both cases.

Figures $2-5$ show a comparison of the less optimal Einstein universe case with the case (29) to illustrate some of the problems one expects in the evolution of nontrivial spacetimes. Figure 2 shows the time evolution of $h_{x x}$ along the positive $x$-axis versus coordinate time for the Einstein universe case and for the case of (29). Figure 2 compares the corresponding contour lines. While no deviation from staticity is visible for the Einstein universe case, the other case shows a rapidly growing peak in $h_{x x}$ and the lapse (shown in Fig. 4) (and thus of $\operatorname{det} h$ ), which is located in the transition zone outside of $\mathscr{I}^{+}$. Eventually this feature cannot be resolved any more and the code crashes. In the Einstein 

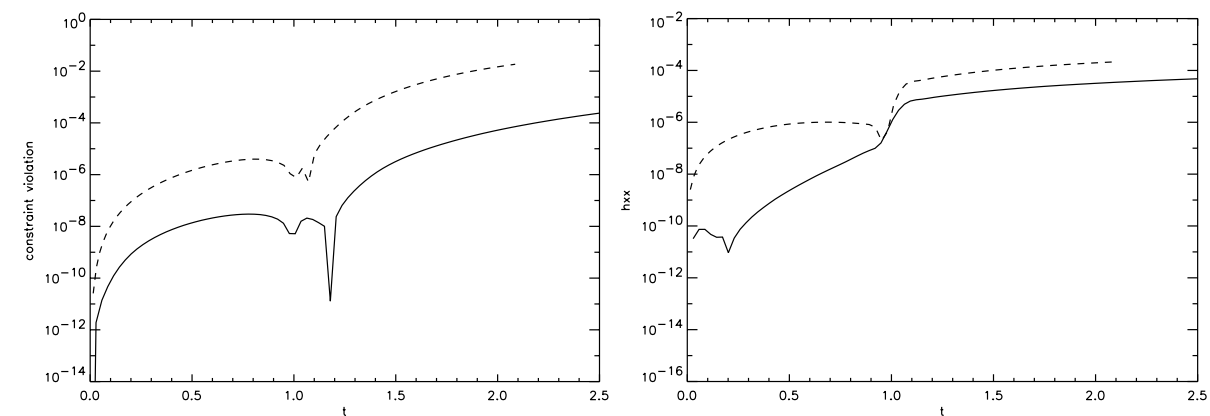

Fig. 1. Comparing the Minkowski (solid line) and Einstein universe (dashed line) cases: left, the value of the constraint $\nabla_{x} \Omega=\Omega_{x}$ at the center is plotted versus coordinate time. In the right image $h_{x x}-1$ is plotted vs. coordinate time (where $t$ is scaled such that $\left.t\left(i^{+}\right)=1\right)$
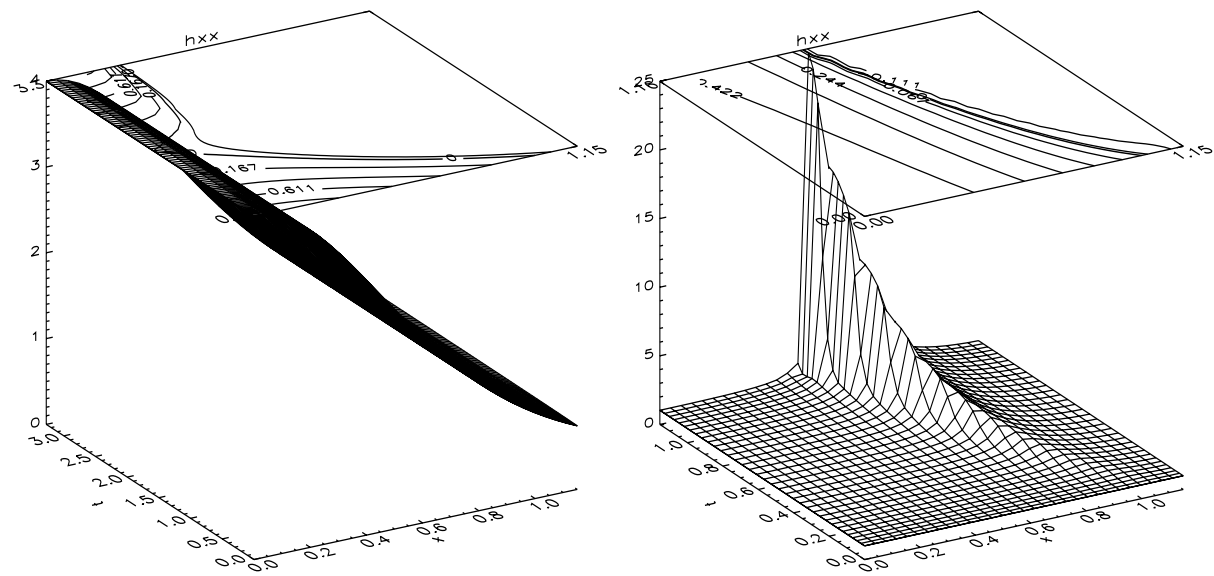

Fig. 2. The value of the metric component $h_{x x}$ for $x \geq 0$ is plotted versus coordinate time. The left image shows the Einstein universe case. The right image shows case (29). There the maximum of $h_{x x}$ in the region where $\Omega>0$ is approximately at the value 5

static case the code was simply stopped by running out of time in the queue. Fig. 5 shows the sum over the $L^{2}$-norms (taken in the physical region) of all the constraints versus time. While in the Einstein static case the constraints show a rapid decrease in the physical region, followed by a steep growth after passing through $i^{+}$, the case (29) exhibits roughly exponential overall growth almost from the start.

Results for the completely static gauge given by (37) are shown in Figs. 6 - 9 . This gauge poses a harder challenge than the previous ones, where $i^{+}$is reached in finite time. Now the goal is to maintain an indefinite stable evolution. However, the evolution shows exponential growth, illustrated in Figs. 8 and 9 by the values of $h_{x x}$ and constraints $\nabla_{x} h_{x x}$ and $\nabla_{x} \Omega=\Omega_{x}$. It is interesting, however, that the curvature invariants $I$ and $J$ are decreasing during the evolution as shown 

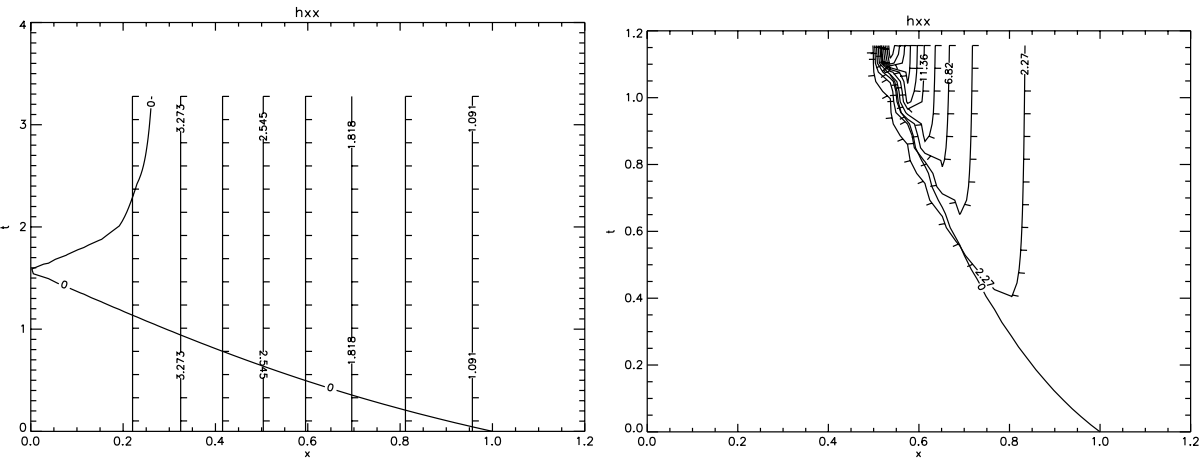

Fig. 3. Contour lines of the metric component $h_{x x}$ for $x \geq 0$ are plotted versus coordinate time. The left image shows the Einstein universe case. The right image shows case (29). The thicker line marks $\Omega=0$, i.e. $\mathscr{I}^{+}$
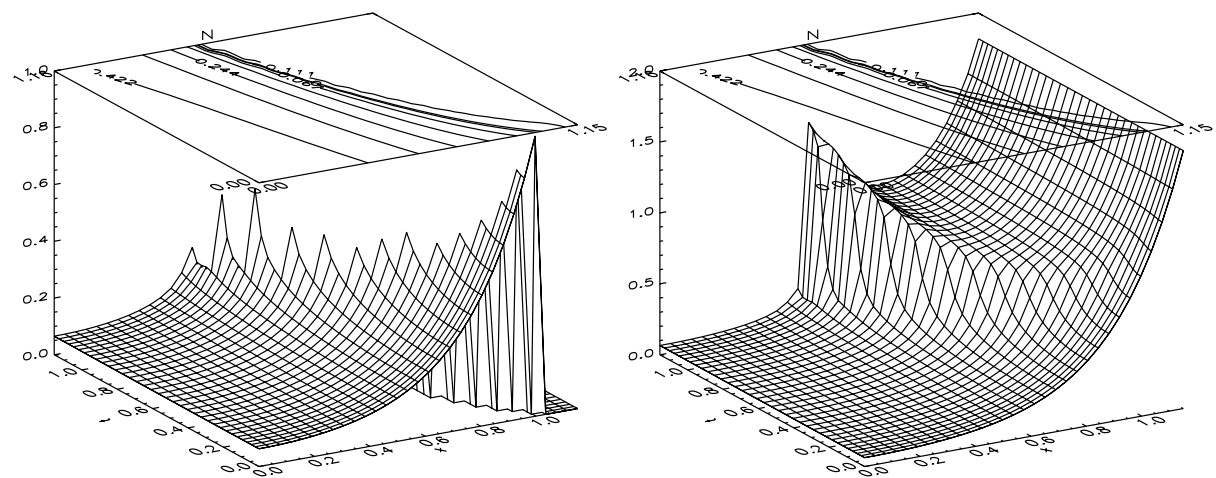

Fig. 4. The value of the lapse $N$ for $x \geq 0$ is plotted for the case (29) versus coordinate time. The left image shows the points where $\Omega>0$. The right image shows all points
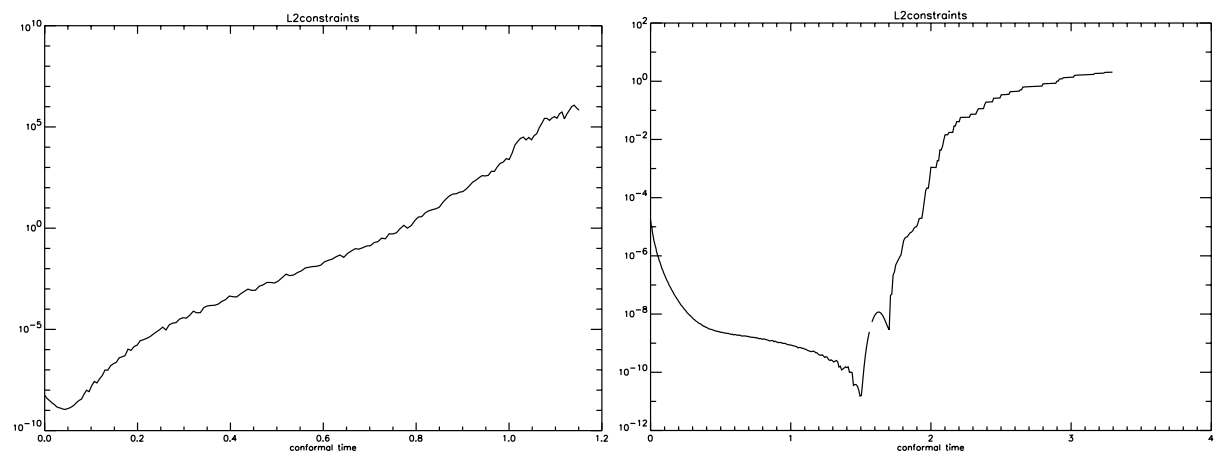

Fig. 5. The sum over the $L^{2}$-norms (taken in the physical region) of all the constraints is plotted versus coordinate time for the Einstein universe (left) and case (29) (right) 
in Figs. 6 and 7. The exponential blowup crashes the code at $t \sim 5.1$. This time seems to be roughly independent of resolution, size of time step, amount of dissipation, location of the boundary and location of the transition zone. A possible explanation is exponentially growing constraint violating modes on the continuum level.
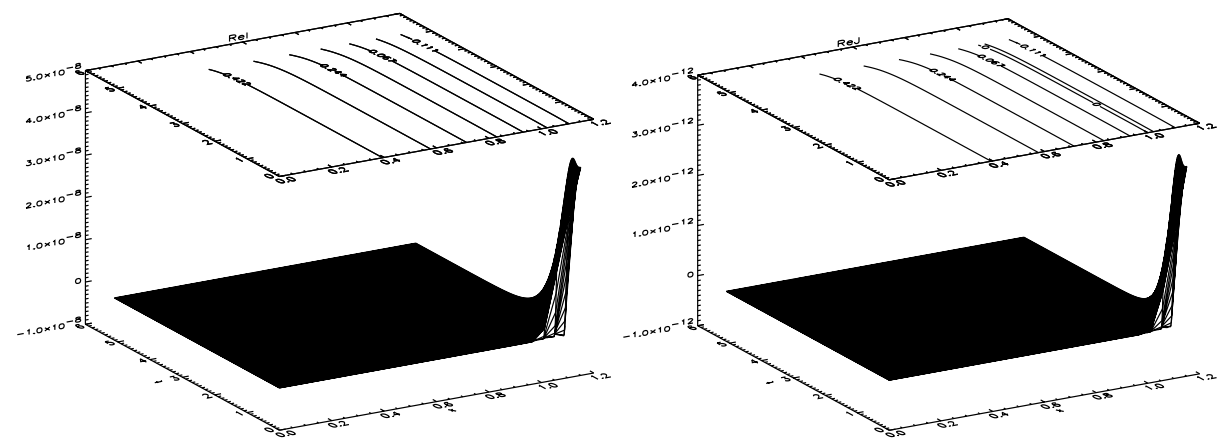

Fig. 6. The real parts of the curvature invariants $I$ (left) and $J$ (right) for $x \geq 0$ are plotted versus coordinate time for the static gauge of (37). Superimposed are contour lines of the conformal factor $\Omega$
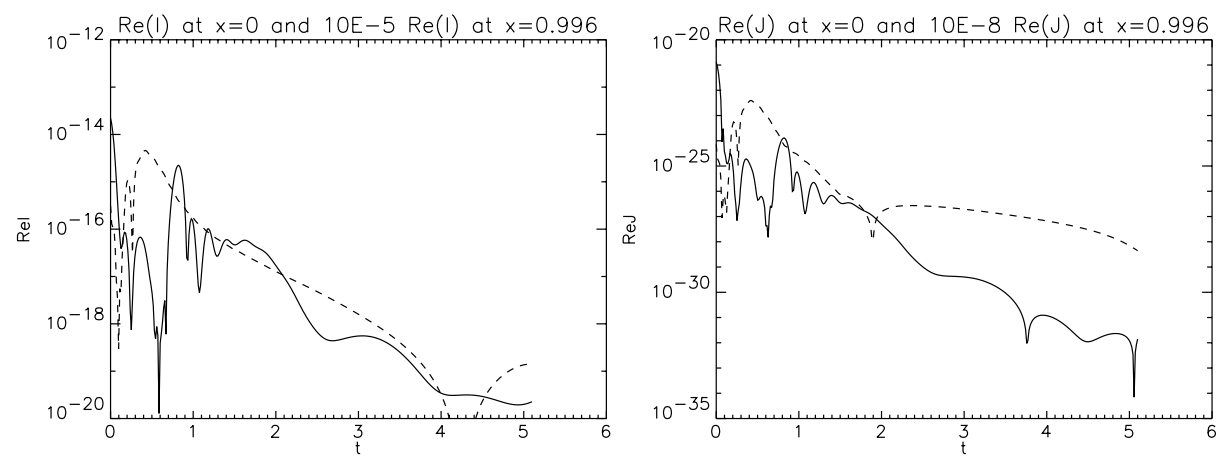

Fig. 7. The real parts of the curvature invariants $I$ (left) and $J$ (right) are plotted versus coordinate time for the static gauge of (37). The solid line is for the gridpoint at the center of the grid, the dashed line for a grid point at $x=0.996, y=z=0$, multiplied by a factor of $10^{-5}$ for $I$ and $10^{-8}$ for $J$

\section{2 "Brill" Data}

We use an axisymmetric Brill-wave type ansatz to look at initial data that contain radiation and set

$$
\mathrm{d} s^{2}=\omega^{2}\left(\mathrm{e}^{2 Q}\left(\mathrm{~d} \varrho^{2}+\mathrm{d} z^{2}\right)+\varrho^{2} \mathrm{~d} \varphi^{2}\right),
$$



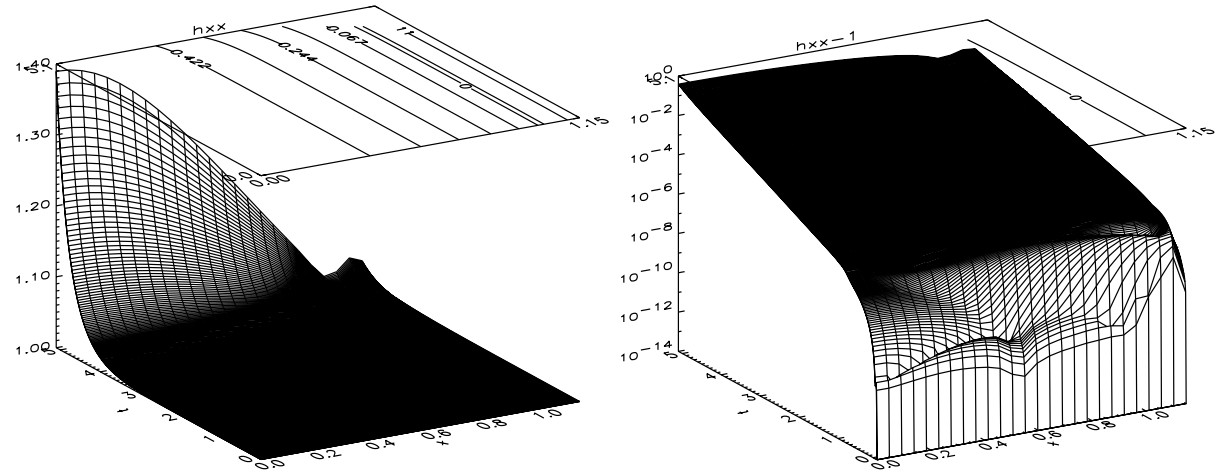

Fig. 8. The value of the metric component $h_{x x}$ for $x \geq 0$ is plotted versus coordinate time with linear (left) and logarithmic (right) scaling for the static gauge of (37). Approximately exponential growth is obvious. The largest amplitude of the growth is in the center

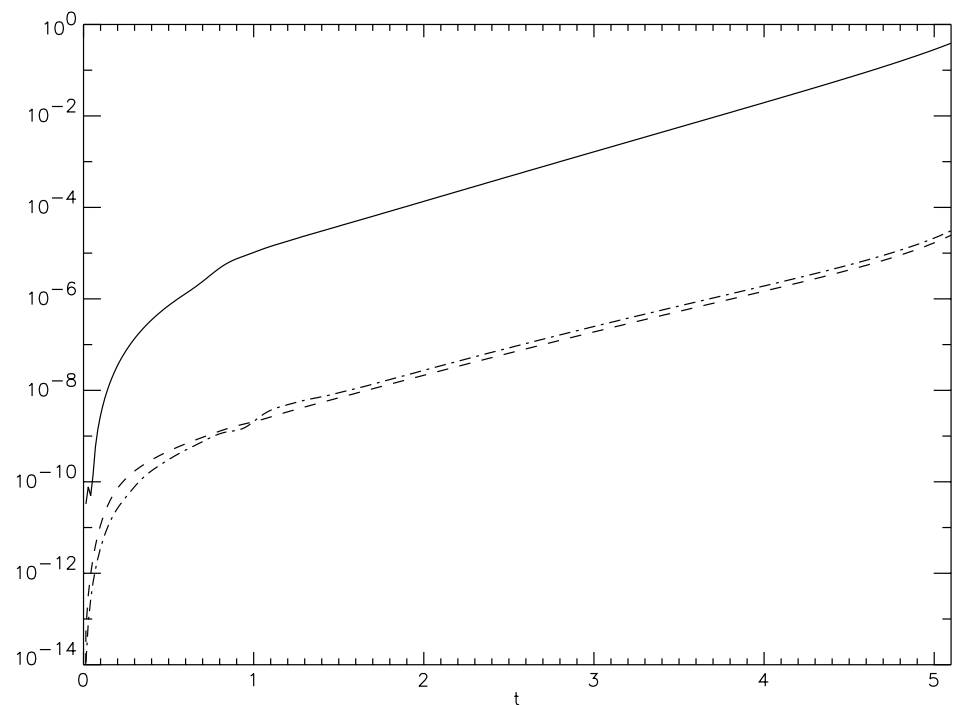

Fig. 9. The values of $h_{x x}$ (solid line) and the constraints $\nabla_{x} h_{x x}$ (dot-dashed) and $\nabla_{x} \Omega=\Omega_{x}$ (dashed) are plotted versus coordinate time for the static gauge of (37)

where $\varrho^{2}=x^{2}+y^{2}$. With $Q=\ln \left(1+A \bar{\Omega}^{2} \varrho^{2} f\left(\varrho^{2}\right)\right) / 2$, in Cartesian coordinates the conformal three-metric becomes

$$
h_{\mathrm{B}}=\omega^{2}\left(\begin{array}{ccc}
1+A x^{2} \bar{\Omega}^{2} f & A x y \bar{\Omega}^{2} f & 0 \\
A x y \bar{\Omega}^{2} f & A y^{2} \bar{\Omega}^{2} f & 0 \\
0 & 0 & 1+A \bar{\Omega}^{2} f
\end{array}\right) .
$$

The axial symmetry makes it easier to analyze the data and choose the gauges. Here we set $\omega=f=1$ and $A=1$. 
Figure 10 shows the real part of the physical curvature invariant $\tilde{I}=\Omega^{6} I$ and the mass loss $\dot{M}_{\mathrm{B}}$. The curvature invariant $\tilde{I}$ is computed both as a perturbation of the Einstein universe and case (29) (triangles) for a "Brill wave" with $A=1$, to demonstrate that the physical initial data are indeed identical. The mass loss $\dot{M}_{\mathrm{B}}$ is computed as a perturbation of the Einstein static case $\left(R_{g}=6\right)$ and plotted in a logarithmic scale. Note that the falloff levels off at late times to a constant value due to numerical error. Note also that oscillations, as we show here, are absent from the initial data corresponding to (44) as shown in Fig. 5 of $[46]$.
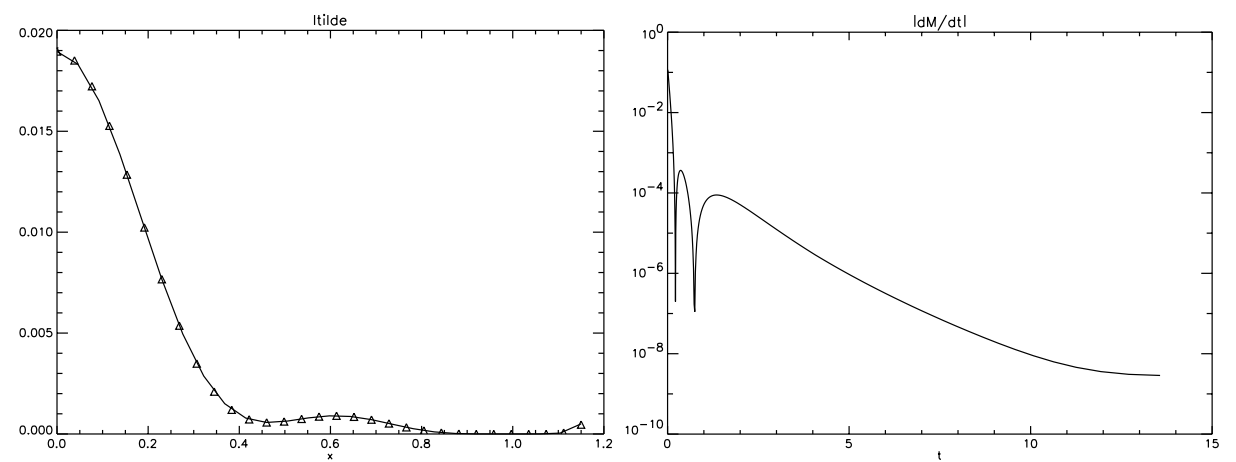

Fig. 10. The left image shows the real part of the physical curvature invariant $\tilde{I}=\Omega^{6} I$, computed as a perturbation of the Einstein universe (line) and case (29) (triangles) for a "Brill wave" with $A=1$. The right image shows the corresponding mass loss function $\dot{M}_{\mathrm{B}}$, computed as as a perturbation of the Einstein static case $\left(R_{g}=6\right)$

\section{Conclusions and Outlook}

Bringing the conformal approach to numerical relativity to full fruition such that it can be used as a tool to explore new physics - in particular in black hole spacetimes - will be a long term effort. In order to contemplate the scope of this project, let us give a drastically oversimplified definition of the art of numerical relativity as a procedural recipe:

1. Find a well posed formulation of the initial(-boundary) value and initial data (constraint) problems for general relativity (optimally, well-posedness should be a theorem but good numerical evidence may be considered sufficient).

2. Without destroying well-posedness, modify your equations and choose your gauges, such that your problem actually becomes well-conditioned ${ }^{5}$.

3. Construct a solid numerical implementation, flexible enough to handle experiments as required by science and by finding solutions to the problems associated with point two.

${ }^{5}$ Ill conditioned problems are those where a result depends very strongly on input, i.e. on initial data, see e.g. Sect. 1.6 and 6.1 of [52]. 
4. Discover (new) results in physics.

5. Explain what you achieved (and how) to fellow numerical relativists and others, such as mathematical relativists, astrophysicists, cosmologists, or mathematicians.

Even without considering the last point (which the present article humbly tries to serve), numerical relativity is a challenging enterprise.

The conformal approach complies with point one in the optimal sense: the equations are regular in the whole spacetime, including the asymptotic region, there are no ambiguities associated with ad-hoc cutoffs at finite distance and the evolution equations are symmetric hyperbolic, which guarantees well-posedness of the initial value problem and allows well-posed initial-value-boundary problems.

Point two, however, already poses a significant challenge: well-defined is not well-conditioned, well-posed problems may still be hopelessly ill-conditioned for numerical simulation. A simple example is provided by any chaotic dynamical system (in the sense of ordinary differential equations). When it comes to solving the Einstein equations, the gauge freedom of the theory results in having more equations (constraints and evolution equations) than variables and more variables than physical degrees of freedom. This redundancy can easily lead to spurious approximate solutions. Different ways to write the equations are only equivalent with regard to exact solutions, but approximations will tend to exhibit constraint violating or gauge modes that may grow very fast (e.g. exponentially). This is perfectly consistent with well-posedness but not acceptable numerically. Even without triggering instabilities, the choice of a bad gauge is likely to create features in the solution which are in practice impossible to resolve. The "good news" is that many of the problems encountered with the conformal field equations have counterparts in traditional approaches to numerical relativity. The way toward solving these problems usually takes the form of gaining insight from simplifications and analytical studies, which then have to be tested in full numerical simulations. This requires a flexible code that is geared toward performing the necessary experiments, which leads to point three - another hard task for classical relativists, because it requires an engineering attitude many relativists are not familiar with. The gauge freedom of general relativity and absence of a natural background creates an additional twist when it comes to point four, which leads to numerous technical and conceptual subtleties.

What is the roadmap for the future? In order to comply with points two and three of the above recipe, preliminary work is carried out toward a new 3D code that will be flexible enough to carry out a range of numerical experiments in order to come up with well-conditioned algorithms for the conformal field equations. One major issue in the improvement of algorithms is to implement a better boundary condition which does not require a transition zone, allows the boundary to be closer to $\mathscr{I}^{+}$and minimize constraint violations generated at the boundary or outside $\mathscr{I}$. Here an essential problem is that $\mathscr{I}^{+}$has spherical cuts and algorithms based on Cartesian grids are probably not optimal. Certainly, a lot of energy will have to be devoted to the question of finding appropriate 
gauge conditions. Particularly hard seems to be the question of how to choose the Ricci scalar $R$ of the unphysical spacetime. Since $R$ steers the conformal factor implicitly through nonlinear PDEs, it seems very hard to influence the conformal factor in any desired way.

An important role in improving the analytical understanding and in setting up numerical experiments will be played by the utilization of simplifications. Particularly important are spacetime symmetries and perturbative studies. Minkowski and Kruskal spacetimes provide particularly important cases to be studied in this context. An alternative route to simplification, which has been very successful in numerical relativity, is perturbative analysis, e.g. with Minkowski or Schwarzschild backgrounds. In the context of compactification this has been carried out numerically with characteristic codes in [53,54] (using appropriate variables in the Teukolsky equations, the perturbation equations are made regular at $\mathscr{I}^{+}$). Some of the problems that showed up there are likely to be relevant also for the conformal approach.

The theory of general relativity is known as a never drying out source for subtle questions in physics and mathematics. Numerical relativity is hoped to help answer some important questions - but at the same time poses many new ones. Without a thorough understanding of how to obtain approximate solutions, our insight into the theory seems incomplete. For isolated systems, the mastering of compactification techniques promises reliability and precision. The next years are expected to see some significant progress in this direction.

\section{Acknowledgments}

The author thanks R. Beig, J. Frauendiener, H. Friedrich, B. Schmidt, and M. Weaver for helpful discussions, C. Lechner and J. Valiente Kroon for a careful reading of the manuscript, and $\mathrm{P}$. Hübner and $\mathrm{M}$. Weaver for letting him use their codes, explaining their results and providing general support in order to take over this project.

\section{References}

1. M. Choptuik: Phys. Rev. Lett. 70, 9 (1993)

2. B. Kleihaus, J. Kunz: Phys. Rev. Lett. 78, 2527 (1997), Phys. Rev. Lett. 791595 (1997)

3. A. Rendall, M. Weaver: Class. Quantum Grav. 18, 2959 (2001)

4. R. Penrose: Phys. Rev. Lett. 10, 66 (1963)

5. H. Friedrich, Proc. R. Soc. A375, 169 (1981)

6. H. Friedrich, Proc. R. Soc. A378, 401 (1981)

7. H. Friedrich, Commun. Math. Phys. 91, 445 (1993)

8. H. Friedrich, Journal of Geometry and Physics 24, 83 (1998)

9. H. Friedrich: gr-qc/0201006

10. P. Hübner, Black Class. Quantum Grav. 16, 2145 (1999)

11. P. Hübner, Equation Class. Quantum Grav. 16, 2823 (1999)

12. P. Hübner, Class. Quantum Grav. 18, 1421 (2001) 
13. P. Hübner, Class. Quantum Grav. 18, 1871 (2001)

14. E. T. Newman, K. P. Tod: 'Asymptotically Flat Space-Times'. In: General Relativity and Gravitation. ed. by A. Held (Plenum Press, New York, 1980) pp. 1-36.

15. R. Wald: General Relativity (University of Chicago Press, Chicago, 1984)

16. J. Stewart: Advanced General Relativity (Cambridge University Press, Cambridge, 1991)

17. J. Frauendiener: Living Rev. Rel. 4, 1 (2000)

18. R. Penrose: Proc. R. Soc. A284 159 (1965)

19. R. Arnowitt, S. Deser, C. W. Misner: 'The dynamics of general relativity'. In: Gravitation: An Introduction to Current Research. ed. by L. Witten (Wiley, New York, 1962) pp. 227-265

20. H. Bondi, M. van der Burg, A. Metzner: Proc. R. Soc. A269, 21 (1962)

21. R. Schoen, S. T. Yau: Commun. Math. Phys. 65, 54 (1979)

22. G. Horowitz, M. Perry: Phys. Rev. Lett. 48, 371 (1982)

23. H. Friedrich, 'Einstein's Equation and Geometric Asymptotics'. In: Proceedings of the GR-15 conference. ed. by N. Dadhich, J. Narlikar (IUCAA, Pune, 1998)

24. J. Frauendiener: Class. Quantum Grav. 17, 373 (2000)

25. P. Hübner, M. Weaver: unpublished

26. J. Frauendiener: Phys. Rev. D58, 064003 (1998)

27. J. Frauendiener: Phys. Rev. D58, 064002 (1998)

28. A. Butscher: In: Proceedings of the International Workshop on the Conformal Structure of Spacetime. ed. by J. Frauendiener, H. Friedrich (Springer Verlag, Lecture Notes of Physics series, to be published)

29. L. Andersson, P. T. Chruściel, H. Friedrich: Comm. Math. Phys. 149, 587 (1992)

30. J. Frauendiener: J. Comput. Appl. Math. 109, 475 (1999)

31. V. Moncrief: In: Workshop on Mathematical Issues in Numerical Relativity http://online.itp.ucsb.edu/online/numrel00/moncrief/ (ITP, Santa Barbara January $10-28$ th, 2000)

32. P. Hübner: Numerische und analytische Untersuchungen von (singulären) asymptotisch flachen Raumzeiten mit konformen Techniken. PhD thesis, LudwigMaximilians-Universität, München, (1993)

33. P. Hübner, Phys. Rev. D53, 701 (1996)

34. M. Pürrer. Diploma thesis, University of Vienna, Vienna, in preparation

35. B. Schmidt: Class. Quantum Grav. 132811 (1996)

36. T. Foertsch: Spacetimes Admitting Null Infinities with Toroidal Sections. Diploma thesis, Technical University of Berlin, Berlin (1997)

37. J. Ehlers, W. Kundt, 'Exact solutions of the gravitational field equations' In: Gravitation: an introduction to current research. ed. by L. Witten (J. Wiley, New York, 1962)

38. P. Hübner, Class. Quantum Grav. 15, L21 (1998)

39. R. Penrose, W. Rindler: Spinors and Spacetime, volume 2 (Cambridge University Press, Cambridge, 1986)

40. L. N. Trefethen: SIAM Review, 24, 113 (1982)

41. M. Hein: Numerische Simulation axialsymmetrischer, isolierter Systeme in der Allgemeinen Relativitätstheorie. Diploma thesis, University of Tübingen, Tübingen (2002)

42. M. Alcubierre, S. Brandt, B. Bruegmann, D. Holz, E. Seidel, R. Takahashi, J. Thornburg: Int. J. Mod. Phys. D10, 273 (2001)

43. B. Gustafsson, H.-O. Kreiss, J. Oliger: Time Dependent Problems and Difference Methods, (Wiley, New York, 1995) 
44. M. Weaver: private communication

45. H. Friedrich: Commun. Math. Phys. 107, 587 (1986)

46. S. Husa: Proceedings of the International Workshop on the Conformal Structure of Spacetime. ed. by J. Frauendiener, H. Friedrich (Springer Verlag, Lecture Notes of Physics series, to be published)

47. M. Alcubierre, B. Brügmann: Phys. Rev. D63, 104006 (2001)

48. B. Schmidt: 'Numerical Evolution of the Kruskal Spacetime Using the Conformal Field Equations'. In: Proceedings of the 20th Texas Symposium on Relativistic Astrophysics. ed. by J. Craig Wheeler, H. Martel (American Institute of Physics, 2001), pp. $729-733$

49. F. Siebel: Simultanes Numerisches Lösen von Zeitentwicklungsgleichungen und Zwangsbedingungen der Einsteinschen Feldgleichungen. Diploma thesis, LMU, Munich (1999)

50. P. Hübner, F. Siebel: Phys. Rev. D64, 024021 (2001)

51. O. Brodbeck, S. Frittelli, P. Hübner, O. Reula: J. Math. Phys. 40, 909 (1999)

52. K. E. Atkinson: An introduction to numerical analysis, 2 nd edn. (Wiley, New York, 1989)

53. M. Campanelli, R. Gomez, S. Husa, J. Winicour, Y. Zlochower: Phys. Rev. D63, $124013(2001)$

54. S. Husa, Y. Zlochower, R. Gomez, J. Winicour: Phys. Rev. D (to be published) 